\title{
Human colon mucosal biofilms from healthy or colon cancer hosts are carcinogenic
}

\author{
Sarah Tomkovich, ${ }^{1}$ Christine M. Dejea, ${ }^{2}$ Kathryn Winglee, ${ }^{3}$ Julia L. Drewes, ${ }^{2}$ Liam Chung, ${ }^{2}$ Franck Housseau, ${ }^{2}$ Jillian L. Pope, ${ }^{1}$ \\ Josee Gauthier, ${ }^{1}$ Xiaolun Sun, ${ }^{1}$ Marcus Mühlbauer, ${ }^{1}$ Xiuli Liu, ${ }^{4}$ Payam Fathi, ${ }^{2}$ Robert A. Anders, ${ }^{5}$ Sepideh Besharati, ${ }^{5}$ \\ Ernesto Perez-Chanona, ${ }^{1}$ Ye Yang, ${ }^{1}$ Hua Ding, ${ }^{2}$ Xinqun Wu, ${ }^{2}$ Shaoguang Wu, ${ }^{2}$ James R. White, ${ }^{6}$ Raad Z. Gharaibeh, ${ }^{1}$ \\ Anthony A. Fodor, ${ }^{3}$ Hao Wang, ${ }^{2}$ Drew M. Pardoll, ${ }^{2}$ Christian Jobin,, ${ }^{1,7}$ and Cynthia L. Sears ${ }^{2}$ \\ 'Department of Medicine, University of Florida, Gainesville, Florida, USA. ${ }^{2}$ Bloomberg-Kimmel Institute for Immunotherapy, Departments of Oncology and Medicine and the Sidney Kimmel Comprehensive \\ Cancer Center, Johns Hopkins School of Medicine, Johns Hopkins Medical Institutions, Baltimore, Maryland, USA. ${ }^{3}$ Department of Bioinformatics and Genomics, University of North Carolina at Charlotte, \\ Charlotte, North Carolina, USA. “Department of Pathology, Immunology and Laboratory Medicine, University of Florida, Cainesville, Florida, USA. ${ }^{5}$ Department of Pathology, Johns Hopkins University, \\ Baltimore, Maryland, USA. ${ }^{6}$ Resphera Biosciences, Baltimore, Maryland, USA. ${ }^{7}$ Department of Infectious Diseases and Immunology, University of Florida, Gainesville, Florida, USA.
}

\begin{abstract}
Mucus-invasive bacterial biofilms are identified on the colon mucosa of approximately $50 \%$ of colorectal cancer (CRC) patients and approximately $13 \%$ of healthy subjects. Here, we test the hypothesis that human colon biofilms comprise microbial communities that are carcinogenic in CRC mouse models. Homogenates of human biofilm-positive colon mucosa were prepared from tumor patients (tumor and paired normal tissues from surgical resections) or biofilm-positive biopsies from healthy individuals undergoing screening colonoscopy; homogenates of biofilm-negative colon biopsies from healthy individuals undergoing screening colonoscopy served as controls. After 12 weeks, biofilm-positive, but not biofilmnegative, human colon mucosal homogenates induced colon tumor formation in $\mathbf{3}$ mouse colon tumor models (germ-free $\mathrm{Apc}^{\mathrm{Min} 1850 /+} ; / 110^{-/-}$or $\mathrm{Apc}^{\mathrm{Min} 1850 /+}$ and specific pathogen-free $\mathrm{Apc}^{\mathrm{Min} 4716 /+}$ mice). Remarkably, biofilm-positive communities from healthy colonoscopy biopsies induced colon inflammation and tumors similarly to biofilm-positive tumor tissues. By 1 week, biofilm-positive human tumor homogenates, but not healthy biopsies, displayed consistent bacterial mucus invasion and biofilm formation in mouse colons. 16S rRNA gene sequencing and RNA-Seq analyses identified compositional and functional microbiota differences between mice colonized with biofilm-positive and biofilm-negative communities. These results suggest human colon mucosal biofilms, whether from tumor hosts or healthy individuals undergoing screening colonoscopy, are carcinogenic in murine models of CRC.
\end{abstract}

\section{Introduction}

The intestinal microbiome has been implicated in a wide variety of diseases, spanning from metabolic, neurologic, and cardiovascular conditions to localized intestinal diseases, including colorectal cancer (CRC). In recent years, numerous studies have supported the notion that individual organisms are associated with human CRC and are capable of inducing tumorigenesis in genetically susceptible murine models of disease (1-3). These efforts have led to the identification of single species harboring prooncogenic genes, including enterotoxigenic Bacteroides fragilis (ETBF), expressing the BFT toxin, Escherichia coli, with the pks virulence island, and Fusobacterium nucleatum, expressing the FadA and Fap2 adhesins, among others (4-8). Extensive data also support the idea that CRC is associated with altered microbiota communities and that a subset of bacteria in the complex microbiota consistently associate with CRC (9-11).

Authorship note: ST, CMD, CJ, and CLS contributed equally to this work.

Conflict of interest: CLS reports research support from Bristol Myers Squibb.

Copyright: @ 2019 American Society for Clinical Investigation

Submitted: August 14, 2018; Accepted: February 1, 2019.

Reference information: J Clin Invest. 2019;129(4):1699-1712.

https://doi.org/10.1172/JCl124196.
However, demonstration of colon carcinogenesis from human fecal microbiota communities is limited $(12,13)$ and has not yet been reported for human colon mucosa. Trillions of diverse microorganisms reside in the human colon in close proximity to the colonic epithelium, separated only by a protective mucus layer comprising largely MUC2 mucins (14). We have previously shown that the mucus layer of the majority of individuals with tumors proximal to the hepatic flexure of the colon, and a subset of individuals with tumors distal to the hepatic flexure, harbors polymicrobial biofilms in direct contact with epithelial cells on tumor and paired normal tissues from these tumor hosts $(11,15)$. Further, approximately $13 \%$ of healthy subjects undergoing routine screening colonoscopy harbored mucosal biofilms. These multispecies biofilm communities, whether in cancer patients or healthy hosts, correlate with prooncogenic biological changes, namely loss of E-cadherin, increased IL-6, activation of Stat3, increased polyamine synthesis, and increased epithelial proliferation $(15,16)$. Sequencing analysis targeting the V3-V5 region of 16S rRNA did not distinguish genus-level differences between biofilm and nonbiofilm mucosa; however, principal coordinates analysis (PCoA) revealed that biofilm communities from paired normal tissues from cancer hosts align with 
A

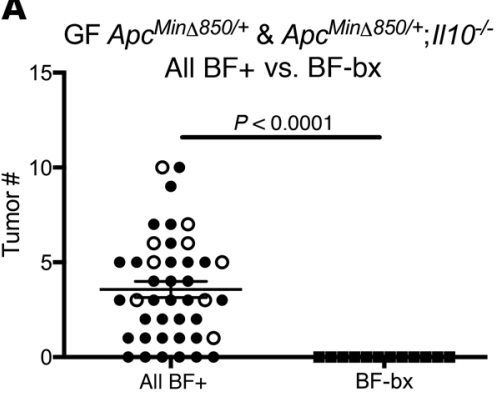

B

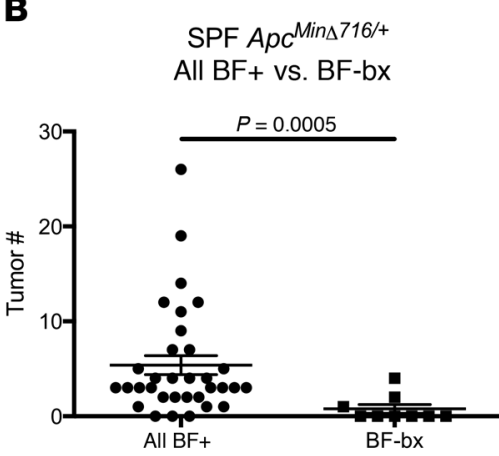

C

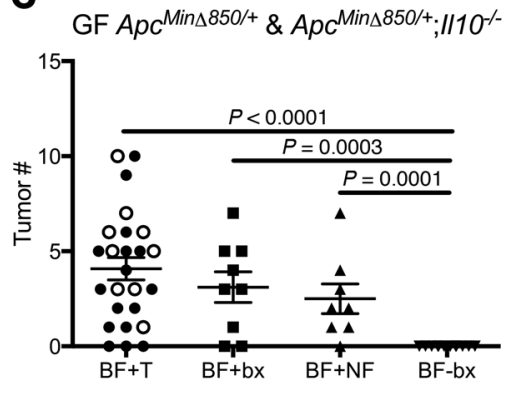

D

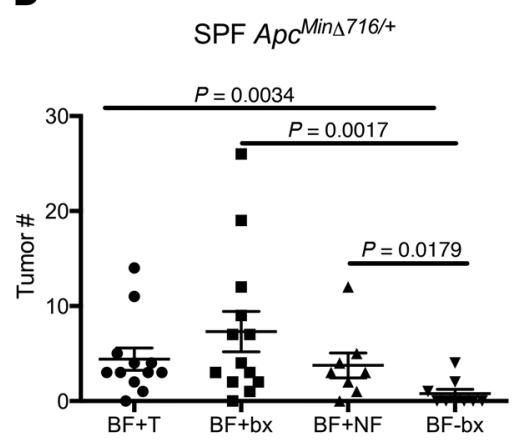

E

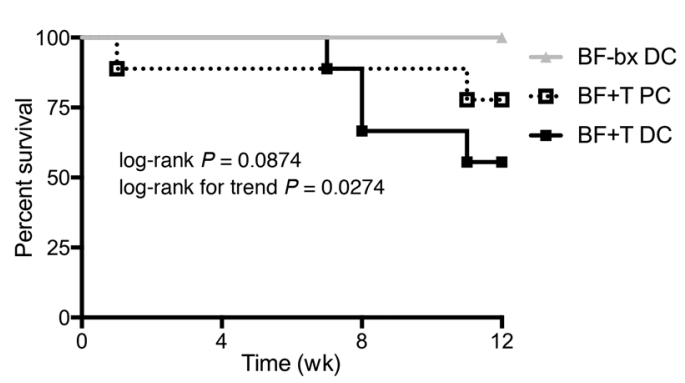

$\mathbf{F}$

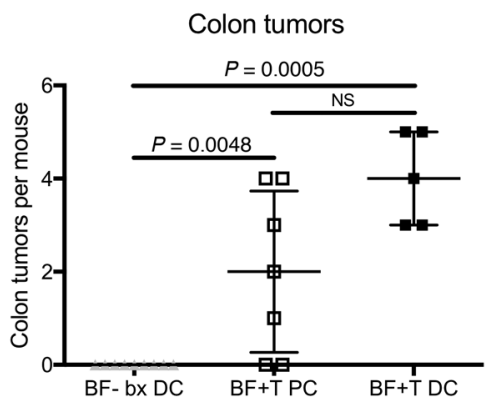

Figure 1. Biofilm-positive human colon tissue inocula are carcinogenic in mouse models. (A and $\mathbf{B})$ Colon tumor counts in $\mathrm{GF}(\mathbf{A}) \mathrm{Apc}^{\mathrm{Min} \triangle 850 /+} ; / 110^{-/-}$and $A p c^{\mathrm{Min} 1850 /+}$ and SPF (B) Apc $c^{\mathrm{Min} 1716 /+}$ mice inoculated with biofilm-positive (BF+) human colon mucosal tissues or biofilm-negative (BF-bx) human colon mucosal tissues. $n=42 \mathrm{BF}^{+}$and $n=12 \mathrm{BF}^{-} A p c^{\text {Min } 1850 /+;} ; / 110^{-/-}$and $A p c^{\text {Min } 1850 /+}$ mice. Black circles represent mice analyzed 12 weeks after inoculation. White circles represent mice harvested 13-20 weeks after inoculation ( $n=9$ mice). $n=33 \mathrm{BF}^{+}$and $n=9 \mathrm{BF}^{-}$for SPF Apc $c^{\text {Minut716/+ }}$ mice. (C and D) Colon tumor counts in GF (C)

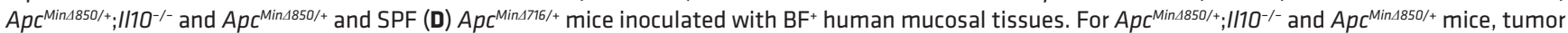
counts from mice inoculated with $\mathrm{BF}^{+}$human tumor (CRC patients) (BF+T, $n=25$ mice), BF+ normal flanking tissues from CRC patients (BF+NF, $n=8$ mice), $\mathrm{BF}^{+}$colonoscopy mucosal biopsies from healthy subjects ( $\mathrm{BF}+\mathrm{bx}, n=9$ mice), and $\mathrm{BF}^{-}$colonoscopy mucosal biopsies from healthy subjects (BF-bx, $n=12$ mice) are displayed. For SPF $A p c^{\text {Min } 4716 /+}$ mice, $n=12(B F+T) ; n=8(B F+N F) ; n=13(B F+b x) ; n=9$ (BF-bx). BF+ conditions do not differ statistically from each other. (E) Survival curve of BF-bx and $\mathrm{BF}+\mathrm{T}$ reassociated $\mathrm{GF} A p c^{\mathrm{Min} 1850 /+} ; / 110^{-/-}$mice over 12 weeks, analyzed by log-rank (Mantel-Cox) test and the log-rank test for trend ( $n=9$ mice per group). Inoculums for reassociation experiments were homogenates of proximal (PC) or distal colon (DC) tissues of mice asso-

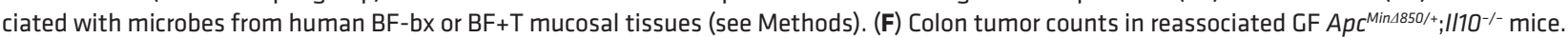
$n=9$ (BF-bx distal colon); $n=7$ (BF+T PC); $n=5$ (BF+T distal colon), since some BF+T reassociated mice did not survive to the 12-week end point. For A-D and $\mathbf{F}$, data are displayed as mean \pm SEM analyzed by Mann-Whitney $U$ test. For $\mathbf{C}, \mathbf{D}$, and $\mathbf{F}, P<0.0167$ is considered statistically significant, based on Bonferroni's correction for multiple comparisons.

tumor-associated communities (15) and are distinct from mucosal communities lacking biofilm formation in either tumor or healthy hosts. This suggests a progressive microbial dysbiosis in the transition from normal tissue to biofilm-covered normal tissue to cancer. These findings raise key and related questions: (a) Are cancer-associated biofilms and their microbial members procarcinogenic or simply associated with cancer as a "bystander"? and (b) Are biofilms and their microbial constituents from cancer-free colons capable of accelerating colon cancer development? In order to determine whether an increase in oncogenic potential would accompany the transition from biofilms on healthy subject mucosa to biofilms on normal mucosa in a CRC host and, finally, to biofilms on tumor tissue from a CRC host, we studied the capacity of biofilm microbial communities to induce CRC upon transfer to both germ-free (GF) and specific pathogen-free (SPF) mice.

\section{Results}

Human colon biofilms are carcinogenic in murine models of disease. To evaluate our hypothesis that biofilm-associated microbial communities have procarcinogenic potential, we utilized 3 murine models of carcinogenesis: (a) GF Apc $c^{\text {Min } 1850 /+}$ (129SvEv),

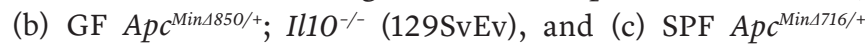
$(\mathrm{C} 57 \mathrm{BL} / 6)$ mice. These models have been used extensively to experimentally test the role of $A p c$ and Wnt signaling, which is critical to the development of human colon cancer, in colon tumorigenesis $(17,18)$. In general, these murine models (when possessing a conventional murine microbiota) develop mostly small intestinal tumors with spare sporadic colon tumors that are predominantly adenomas. The mice most often expire due to bowel obstruction or bleeding prior to developing histologic cancer. Inclusion of $I l 10^{-/}$additionally tested the role of IL-10 signaling, which is considered important in very early onset human inflammatory bowel disease (19).

Mice were inoculated with a mucosal homogenate combined from 5 surgical CRC patients (biofilm-positive cancers or biofilm-positive paired normal tissues) or healthy subjects who underwent colonoscopy (biofilm-positive or biofilm-negative biopsies) (see Methods, Supplemental Table 1, and Supplemental Figure 1; supplemental material available online with this article; https://doi.org/10.1172/JCI124196DS1) to create the following inoculated mouse groups: $\mathrm{BF}+\mathrm{T}, \mathrm{BF}+\mathrm{NF}, \mathrm{BF}+\mathrm{bx}$, and 
A

Invasive adenocarcinoma in GF $A p c^{M i n} \Delta 850 /+; / 10^{-/-}$ mice associated with human $B F+T$ mucosa

$16 \mathrm{wk}$

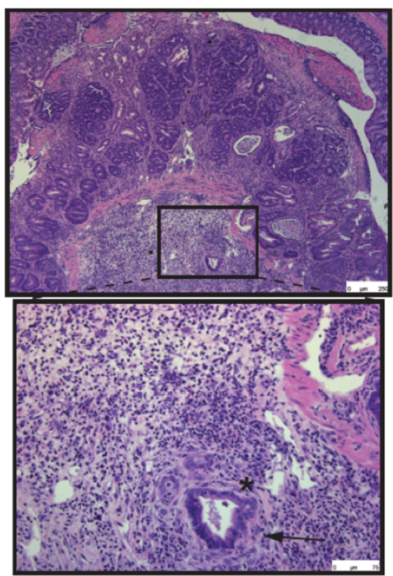

$20 w k$

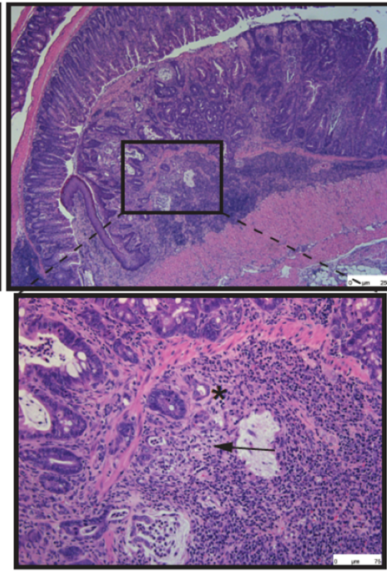

B

SPF Apc ${ }^{\text {Min } \Delta 716 /+}$ after 3 weeks

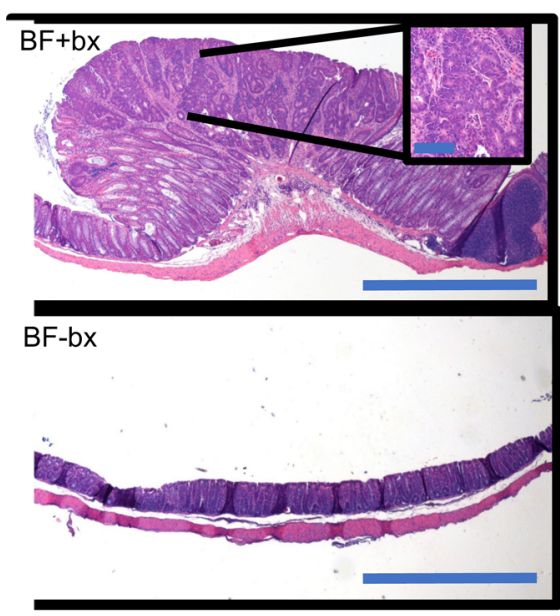

Figure 2. Histopathology of colon tumors from mice associated with human biofilm-positive mucosal tissues. (A) $\mathrm{GF} A p c^{\mathrm{Min} 1850 /+} ; / 110^{-/-}$inoculated with human $\mathrm{BF}+\mathrm{T}$ tissue homogenate develop carcinoma with submucosal invasion after 16 (left panels) or 20 (right panels) weeks. Original magnification, $\times 50$; scale bar: $250 \mu \mathrm{m}$ (upper panels). Bottom panels represent insets of top panel boxed areas demonstrating invasive adenocarcinoma. Original magnification, $\times 200$; scale bar: $75 \mu \mathrm{m}$. In addition to high-grade glandular dysplasia in the mucosa, there were infiltrative glands in the submucosa (asterisks). These submucosal glands also displayed desmoplastic stromal changes (arrows). The overall features were diagnostic of invasive adenocarcinoma. (B) Colon histopathology of SPF Apc $c^{\text {Mind716/+ }}$ mouse inoculated with BF+bx (top panel) or BF-bx (bottom panel) for 3 weeks. Top panel displays a large tumor with multifocal dysplasia and a region of intramucosal adenocarcinoma (inset). Original magnification, $\times 4$; scale bars: 1 mm (upper panel). Original magnification, $\times 20$; scale bars: $0.1 \mathrm{~mm}$ (lower panel).

BF-bx, respectively. In all 3 murine models, inocula prepared from biofilm-covered human mucosa induced colon tumors at 12 weeks after inoculation, primarily in the distal colon, while inocula prepared from biofilm-negative mucosa did not (Figure 1, A-D, and Supplemental Videos 1-4). Remarkably, tumor induction by biofilm communities did not differ by biofilm-positive tissue source. In other words, biofilm communities from the colon biopsies of healthy subjects were equally potent tumor inducers compared with biofilm communities from CRC hosts (CRCs or paired normal tissues) (Figure 1, C and D). Similarly, the murine model

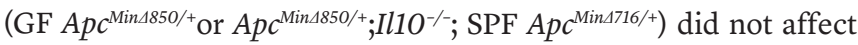
colon tumor induction (Figure 1, C and D), although results were less certain in GF $A p c^{\text {Min } 4850 /+}$ mice, of which smaller numbers were available for study (Supplemental Figure 2). Additional controls that confirmed the importance of the biofilm microbiota communities in these results included the absence of colon tumor formation in GF $A p c^{\operatorname{Min} 4716 /+}$ mice $(n=2)$ after inoculation with heat-inactivated human biofilm-positive tumor tissues and a marked difference in colon tumor formation between GF and SPF $A p c^{\text {Min } \Delta 716 /+} 4$ months of age older (GF, 14 of 15 mice with no colon tumors [median, 0 tumors; range, $0-1$ ] vs. SPF, 6 of 14 mice with no colon tumors [median, 1 tumor; range, $0-5) P<0.0027$, Fisher's exact test and Mann-Whitney $U$ test). Previous work

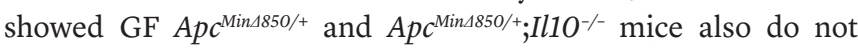
develop colon tumors (20).

To further test the carcinogenicity of the human BF+T microbiota, homogenized mouse proximal or distal colon tissues from initial BF-bx- and BF+T-inoculated GF $\mathrm{Apc}^{\mathrm{Min} \Delta 850 /+} ; \mathrm{IllO}^{-/-}$mice were inoculated into new cohorts of GF $\mathrm{Apc}^{\mathrm{Min} \Delta 850 /+} ; \mathrm{IllO}^{-/-}$mice (reassociation experiment). Several reassociated mice in the
$\mathrm{BF}+\mathrm{T}$ groups died or required euthanasia prior to the 12-week end point (Figure 1E). Nonetheless, $\mathrm{BF}+\mathrm{T}$ reassociated mice displayed significantly increased colon tumors compared with mice reassociated with BF-bx murine tissues, which displayed no colon tumors (Figure 1F). These results show that the human biofilm-positive mucosal microbiota communities that assemble in the mucosa

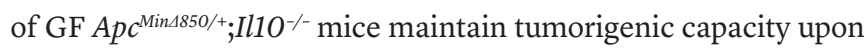
transfer to additional GF mice.

Experiments on a small number of biofilm-positive tumorassociated GF $A p c^{\text {Min } 1850 /+}$ and $A p c^{\text {Min } 1850 /+} ; \mathrm{Il1O}^{-/-}$mice $(n=9)$ were carried out beyond 12 weeks to determine whether the tumors would develop into carcinomas; 2 mice (22\%) developed invasive adenocarcinomas that were characterized by infiltrative glands in the submucosa surrounded by desmoplastic stroma (Figure 2A). Similarly, during the course of our SPF mouse-model experiments, a small group of $A p c^{\text {Min } 4716 /+}$ mice became ill around 3 weeks after receiving biofilm-positive human mucosal inocula and required euthanasia. After initial histopathology revealed colon tumors, additional mouse groups were analyzed at 3 weeks, revealing early tumor formation by histopathology in 11 of 15 mice inoculated with biofilm-positive human tumor colon mucosa, 4 of 6 mice inoculated with biofilm-positive colonoscopy biopsy mucosa, 0 of 5 mice inoculated with biofilm-positive paired normal mucosa, and 0 of 7 mice inoculated with biofilm-negative colonoscopy biopsy mucosa $(P=0.0039$ and $P=0.02$, biofilm-positive tumor or biofilm-positive biopsy, respectively, vs. biofilm-negative biopsy; Fisher's exact test, Bonferroni's corrected significant $P$ value $<0.025)$. Histopathology further revealed multifocal dysplasia $(n=4$ mice) and adenocarcinoma ( $n=5$ mice) in mice receiving either biofilm-positive tumor or biofilm-positive colonoscopy biopsy mucosa (Figure 2B). 
A

GF $A p c^{M i n} \Delta 850 /+\& A p c^{M i n} \Delta 850 /+; / 110^{-/}$

GF $A p c^{M i n} \Delta 850 /++A p c^{M i n} \Delta 850 /+; / 110^{-/-}$
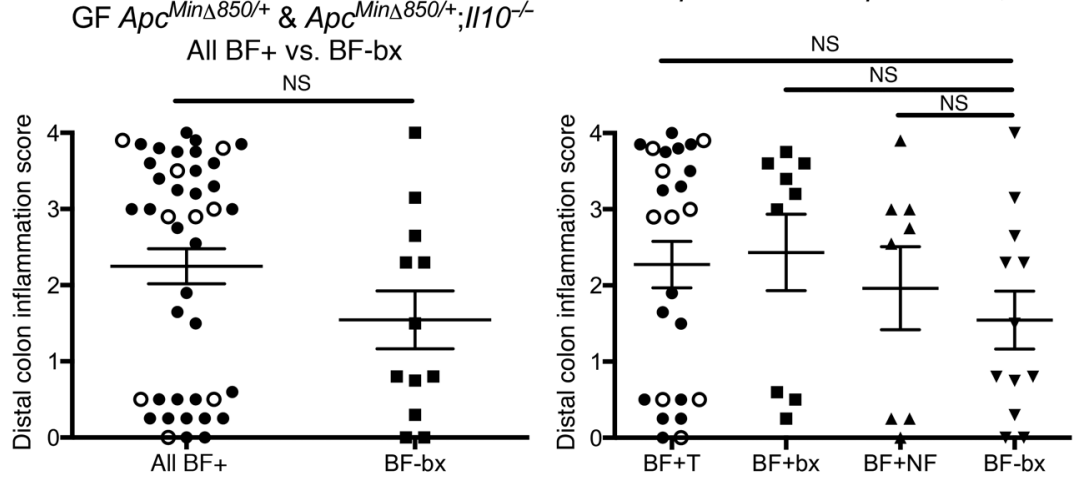

B
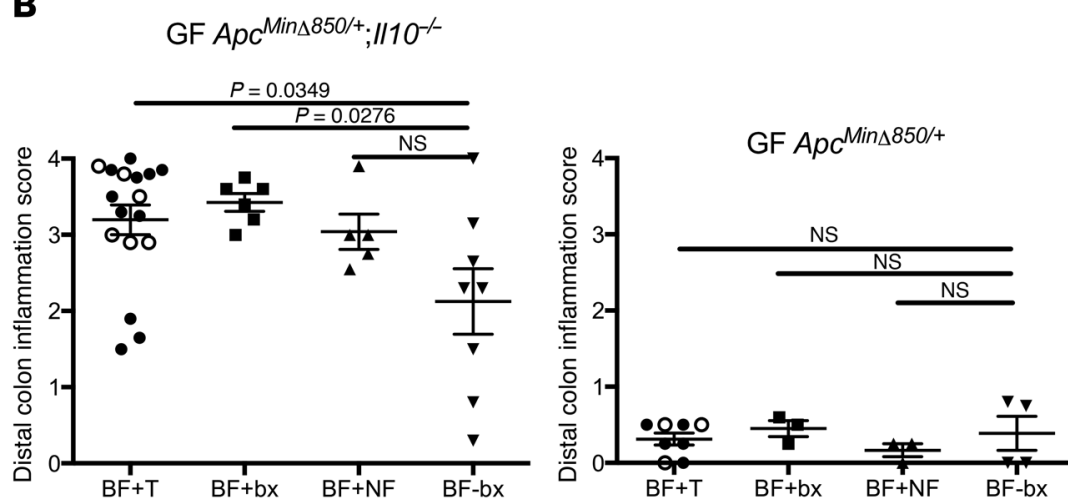

C

GF $A p c^{M i n \Delta 850 /+} \& A p c^{M i n \Delta 850 /+} ; / 110^{-/-}$
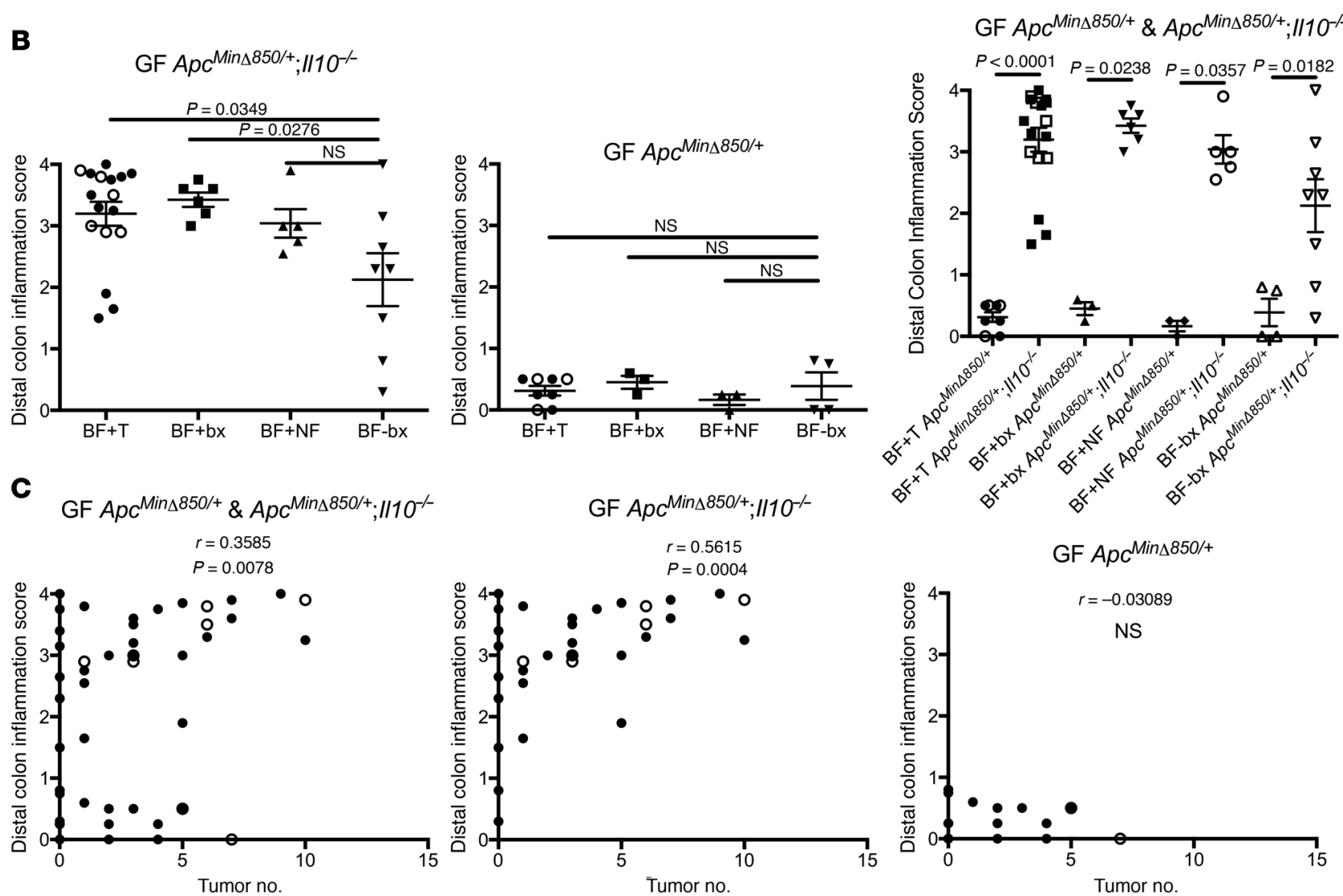

Figure 3. Colon inflammation induced by $\mathrm{BF}^{+}$and $\mathrm{BF}^{-}$human colon mucosal tissue inocula in $\mathrm{GF} \mathrm{Apc}^{\mathrm{Min} 4850 /+} ; / / 10^{-/-}$and $\mathrm{Apc}^{\mathrm{Min} \Delta 850 /+}$ mouse models. (A) Distal colon inflammation scoring (see Methods) for GF Apc $\mathrm{Min} \triangle 850 /+^{\prime} / 1110^{-/-}$and $\mathrm{Apc}^{\mathrm{Min} \Delta 850 /+}$ mice inoculated with $\mathrm{BF}^{+}$or $\mathrm{BF}^{-}$human colon mucosal tissues. Both panels show $\mathrm{BF}^{-}$human colonoscopy biopsies (BF-bx, $n=12$ mice are shown). In the left panel, $3 \mathrm{BF}+\mathrm{groups}(\mathrm{BF}+\mathrm{bx}, \mathrm{BF}+\mathrm{NF}$, and $\mathrm{BF}+\mathrm{T}$ ) are combined into 1 group $\left(\mathrm{BF}^{+}, n=42\right.$ mice), and in the right panel, $\mathrm{BF}^{+}$colon tumor ( $\mathrm{BF}+\mathrm{T}, n=25$ mice), $\mathrm{BF}^{+}$normal flanking tissue from $C R C$ patients $\left(\mathrm{BF}+\mathrm{NF}, n=8\right.$ mice), and $\mathrm{BF}^{+}$colonoscopy biopsies from healthy subjects (BF+bx, $n=9$ mice) are shown separately. Data displayed as mean $\pm \mathrm{SEM}$

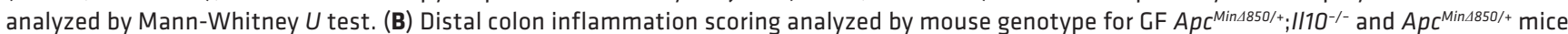

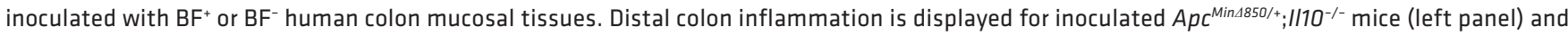
$A p c^{\mathrm{Min} 1850 /+}$ (middle panel) only and is compared across the $A p c^{\mathrm{Min} 1850 /+} ; / 110^{-/-}$and $A p c^{\mathrm{Min} 1850 /+}$ mice genotypes (right panel). Data are displayed as mean \pm SEM and were analyzed by Mann-Whitney $U$ test. For the left panel, $P<0.0167$ was considered statistically significant using Bonferroni's correc-

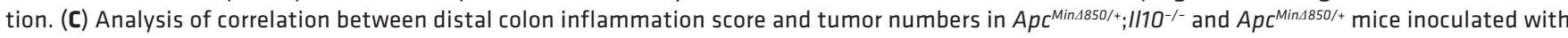
$\mathrm{BF}^{+}$or $\mathrm{BF}^{-}$human colon mucosal tissues. Both mouse genotypes (left panel); $A p c^{\mathrm{Min} 1850 /+} ; / 110^{-/-}$mice only (middle panel); $A p c^{\mathrm{Min} 1850 /+}$ mice only (right

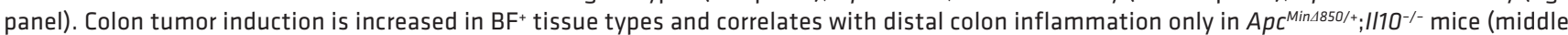
panel). Black circles represent mice analyzed at 12 weeks after inoculation. White circles represent mice harvested at 13-20 weeks after inoculation $(n=9$ mice). Analyzed by Spearman's rank order correlation. 
GF C57BL/6

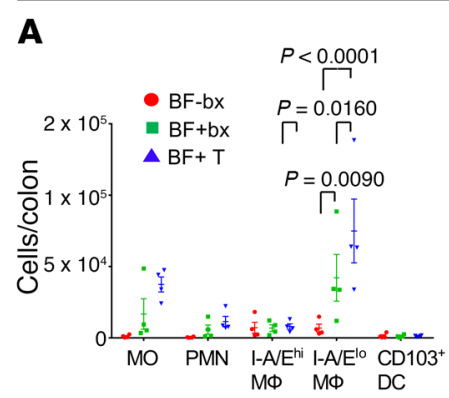

B
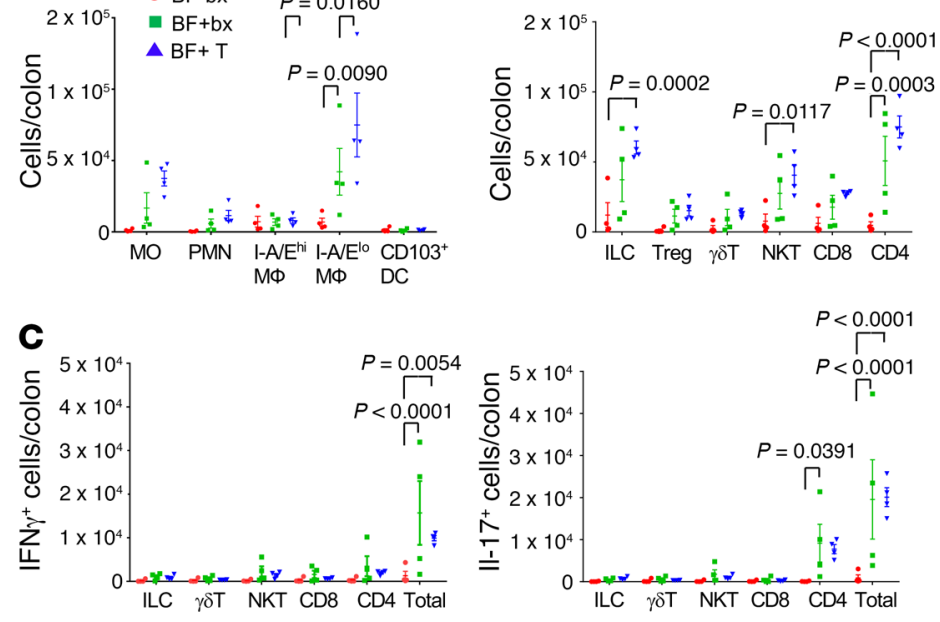

GF $A p c^{\operatorname{Min} \Delta 850 /+}$

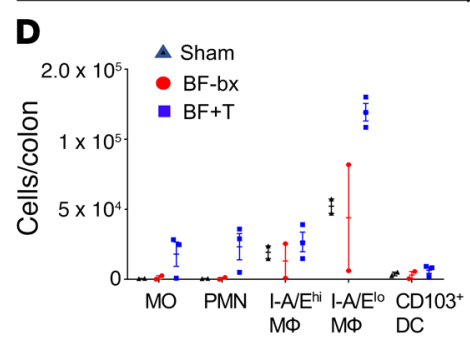

E
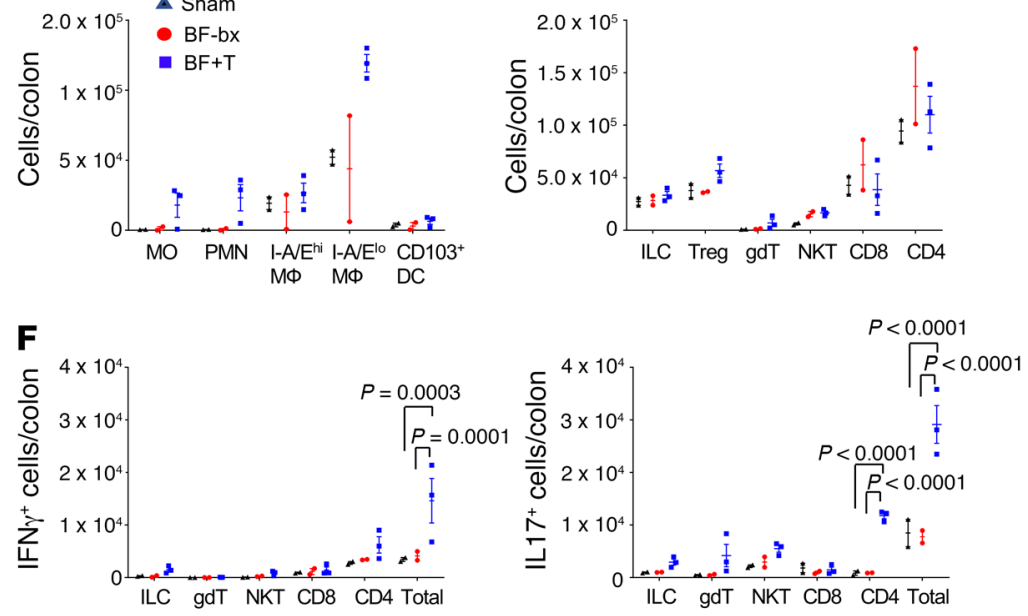

Figure 4. Human $\mathrm{BF}^{+}$colon tissue inocula induce colon inflammation in GF mouse models after 1 week. (A-C) Myeloid (A) and lymphoid (B and C) lamina propria immune cell infiltrates plotted as number of live cells per colon in GF C57BL/6 at 7 days after inoculation with slurries from biofilm-negative colon biopsies (BF-bx, red circles; $n=4$ ), biofilm-positive colon biopsies (BF+bx, green squares; $n=4)$, or biofilm-positive colon tumors (BF+ T, blue triangles; $n=4$ ). In C, number of IFN- $\gamma^{+}$-producing (left graph) and IL-17+-producing (right graph) cells per colon were determined by intracellular staining and flow cytometry analysis. Overall significance across cell types was calculated using 2-way ANOVA with multi-comparison correction (Tukey's test). (D-F) Myeloid (D) and lymphoid (E and F) lamina propria immune cell infiltrates plotted as number of live cells per colon in GF $A p c^{\text {Mind850/- }}$ mice at 7 days after inoculation with buffer (sham control, black triangles; $n=2$ ) or slurries from biofilm-negative colon biopsies (BF-bx, red circles, $n=2$ ) or biofilm-positive colon tumors (BF+T, blue squares; $n=3$ ). In F, number of IFN- $\gamma^{+}$-producing (left graph) and IL-17+-producing (right graph) cells per colon were determined by intracellular staining and flow cytometry analysis. Overall significance across cell types was calculated using 2-way ANOVA with multi-comparison correction (Tukey's test). Data are presented as mean \pm SEM.

Biofilm-positive human colon mucosa inocula also significantly increased small intestine tumor numbers in the GF mouse models, but not the SPF mouse model (Supplemental Figure 3A). Similarly to what occurred with colon tumor induction in the GF $A p c^{M i n \Delta 850 /+}$ and $\mathrm{ApC}^{\mathrm{Min} 485 \mathrm{O}^{++}} ; \mathrm{Il1O}^{-/-}$mouse models, small intestine tumor num- bers did not differ between these 2 mouse models (Supplemental Figure 3B).

Collectively, our murine model results suggest that human colon biofilms - even the biofilms detected in healthy individuals undergoing screening colonoscopy - contain carcinogenic microbes or microbial consortia.

Enhanced inflammation in $\mathrm{ApC}^{\mathrm{Min} 1850 /+} ; \mathrm{Il1O}^{-/-}$ mice is not required for biofilm-mediated tumorigenesis. Overall, whole or distal colon histopathology inflammation scores did not differ between biofilm-positive and biofilm-negative groups and did not differ by tissue source of the inocula (Figure $3 \mathrm{~A}$ and Supplemental Figure 4, A and B). However, the data, particularly for the biofilm-positive inocula, clustered into 2 groups (Figure $3 \mathrm{~A}$ ). The reason for this clustering was resolved by subsequent analyses. Analysis by mouse genotype revealed enhanced whole colon and, in particular, distal colon histologic inflammation in GF $\mathrm{Apc}^{\mathrm{Min} \Delta 850 /+} ; \mathrm{IllO}^{-/-}$when compared with GF $A p c^{\text {Min } 4850 /+}$ mice with functional IL-10 (Figure 3B and Supplemental Figure 4C), consistent with previous studies showing colitis in $\mathrm{Apc}^{\mathrm{Min} \triangle 850 /+} ; \mathrm{IllO}^{-/-}$mice (20). Within the GF $\mathrm{ApC}^{\mathrm{Min} 4850 /+} ; \mathrm{IllO}^{-/-}$mice, distal colon histologic inflammation showed a trend toward higher inflammation in the mice inoculated with biofilm-positive human mucosal tissues compared with mice inoculated with biofilm-negative colonoscopy biopsies (Figure 3B). In contrast, within the GF $A p c^{\text {Min } 1850 /+}$ mice, distal colon histologic inflammation was limited across inocula types (Figure 3B).

We next examined the relationship between detection of inflammation and number of colon tumors induced in the mice. Tumor numbers and distal colon inflammation were positively, but modestly, correlated in $\mathrm{Apc}^{\mathrm{Min} \Delta 850 /+} ; \mathrm{IllO}^{-/-}(r=0.5615$, $P=0.0004)$, but not $A p c^{\mathrm{Min} \Delta 850 /+}$, mice (Figure 3C). Thus, human colon mucosal biofilm-induced murine colon carcinogenesis was independent of histologic colon inflammation in GF $A p c^{\text {Min } 4850 /+}$, but not $\mathrm{Apc}^{\mathrm{Min} \Delta 850 /+} ; \mathrm{Il}^{-/-}$, mice. Collectively, these results suggest that human colon mucosal biofilms may contain microbiota species capable of tumor initiation, as detected in $A p c^{M i n \Delta 850 /+}$ mice, whereas in $\mathrm{ApC}^{\mathrm{Min} \Delta 850 /+} ; \mathrm{IllO}^{-/-}$mice, inflammation further augmented tumor initiation and promotion.

Biofilm-positive human mucosal tissues induce early IL-17 and myeloid cell infiltration in GF mice. We have previously shown that inflammation at 1 week in C57BL/6 and $A p c^{\operatorname{Min} 4716 /+}$ mice reflects early mucosal inflammatory changes predictive of contributions to colon tumorigenesis $(21,22)$. Thus, to limit the impact of $I l 10$ deficiency on colon mucosal inflammation and to study the inflammatory response to human biofilm communities in more detail, we next examined the murine colon mucosal inflammatory responses to human biofilm-positive 
A
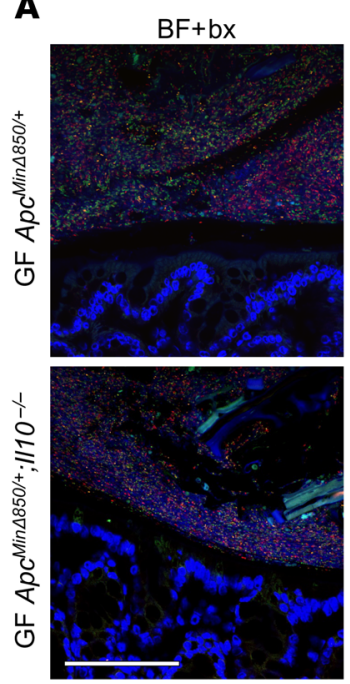

Bacteroidetes Lachnospiraceae Fusobacteria Proteobacteria

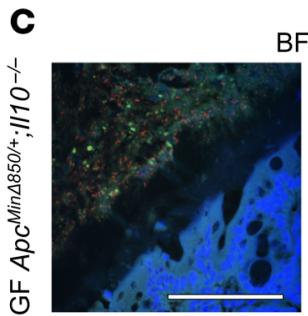

Mouse 1
$\mathrm{BF}+\mathrm{NF}$
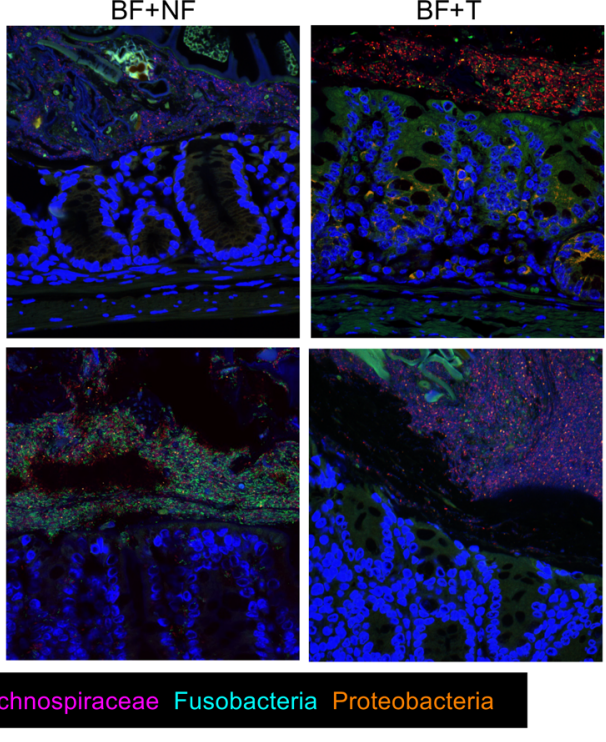

$F-b x$

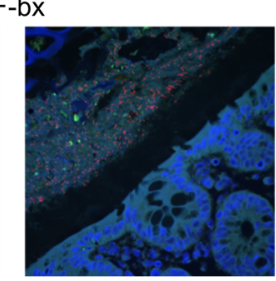

Mouse 2
B

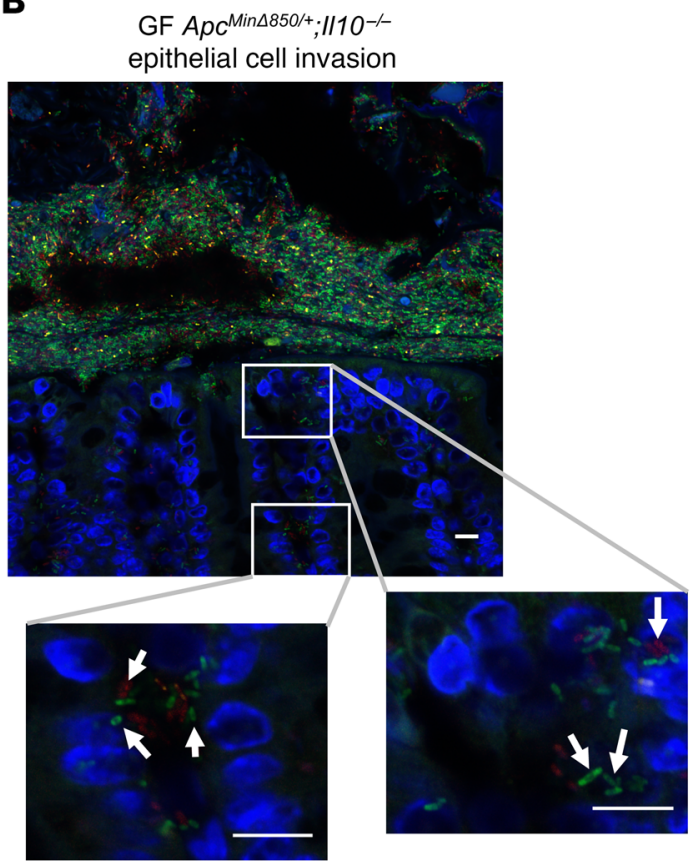

Figure 5. Human BF+ colon tissue inocula induce colon biofilms in mouse models. (A) GF $\mathrm{Apc}^{\mathrm{Min} 4850 /+} ; / / 10^{-/-}$and $\mathrm{Apc} \mathrm{Min}^{\mathrm{M} 850 /+}$ mice 12 weeks after inoculation with $\mathrm{BF}^{+}$human colon mucosal tissue inocula display distal colon biofilm formation except the $\mathrm{BF}+\mathrm{T}_{-}$-inoculated $A p c^{M i n} \Delta 850 /{ }^{+} ; / / 10^{-/-}$mouse. $\mathrm{BF}_{+} \mathrm{T}$, $\mathrm{BF}^{+}$colon tumor; BF+NF, BF+ normal flanking tissue from CRC patients; BF+bx, BF+ colonoscopy biopsy from healthy subjects. $n=1$ mouse per genotype and per human tissue inoculum. Tissues analyzed by multiprobe FISH (see Methods) and counterstained with the nuclear stain DAPI (blue). Corresponding PAS stains are shown in Supplemental Figure 5. Scale bar: $100 \mu \mathrm{m}$. (B) Image from GF Apc ${ }^{\text {Min } \triangle 850 /+}$;/110 ${ }^{-/-}$mouse demonstrating tissue bacterial invasion (arrows). Scale bars: $10 \mu \mathrm{m}$. (C) Two GF Apc ${ }^{\mathrm{Min} \Delta 850 /+} ; / / 10^{-/-}$mice inoculated with BF- colonoscopy biopsies from healthy subjects (BF-bx). Scale bar: $100 \mu \mathrm{m}$. Tissues analyzed by multiprobe FISH (see Methods).

and biofilm-negative communities in GF C57BL/6 mice and GF $A p c^{\text {Min } 1850 /+}$ mice 1 week after inoculation using flow cytometry. Biofilm-negative colon mucosal biopsy inoculum from healthy subjects generally led to low numbers of lymphoid or myeloid mucosal immune cells, identified by flow cytometry analysis in GF C57BL/6 mice (Figure 4). However, inoculation of GF C57BL/6 mice with biofilm-positive colon mucosal biopsy inoculum from healthy subjects or biofilm-positive tumor inoculum induced myeloid cell infiltration, including monocytic (MO) and granulocytic immature myeloid cells (polymorphonuclear cells $[\mathrm{PMN}]$ ) as well as MHC-II ${ }^{\mathrm{lo}}$ macrophages (MФ I-A/E ${ }^{\mathrm{lo}}$ ) (Figure $4 \mathrm{~A}$ ). A prominent $\mathrm{CD}^{+} \mathrm{CD} 4^{+} \mathrm{IL} 17^{+}$mucosal cellular infiltrate (Th17 cells) as well as increased colon infiltration with innate lymphoid cells (ILCs) and NKT cells were observed only following inoculation with biofilm-positive human colon mucosa tissues either from biofilm-positive colonoscopy biopsies or biofilm-positive tumor (Figure 4B). Although total IFN- $\gamma^{+}$ cells were also significantly increased following inoculation of biofilm-positive colonoscopy biopsies or biofilm-positive tumor, total $\mathrm{IL}-17^{+}$cells outnumbered IFN- $\gamma^{+}$cells (Figure $4 \mathrm{C}$ ). Consis-

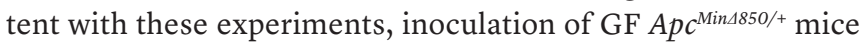

with biofilm-positive tumor, but not biofilm-negative biopsy, human tissues again induced predominant IL-17 mucosal responses and increased myeloid infiltration similarly to that observed in C57BL/6 mice (significant increase of Th17 and total IL-17+ cells; Figure 4, D-F). Thus, despite the lack of histologic inflammation detected at late time points in GF $A p c^{M i n \Delta 850 /+}$ mice, these results suggest that human mucosal biofilm communities increase and likely alter the function of select mucosal immune populations that contribute to colon tumorigenesis, even in

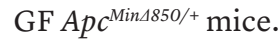

Biofilm-positive human mucosal tumor tissues induce biofilms in GF mice. Whereas the entire length of the human colon is protected by a mucus layer, the protective mucus layer of the murine colon is usually limited to the distal $2-3 \mathrm{~cm}$ of colon $(23,24)$. Furthermore, biofilm formation has only rarely been observed in the murine colon. To see if biofilm formation present in human colon mucosal samples was transmissible after inoculation into GF mice, we first examined, using FISH analyses, the microbial composition and organization on the distal colon mucosa of GF $A p C^{\text {Min } 4850 /+} ; I_{l 1 O^{-/-}}$and $A p c^{\text {Min } 1850 /+}$ mice 12 weeks after inoculation with biofilm-positive (human tumor, paired normal and colonos- 

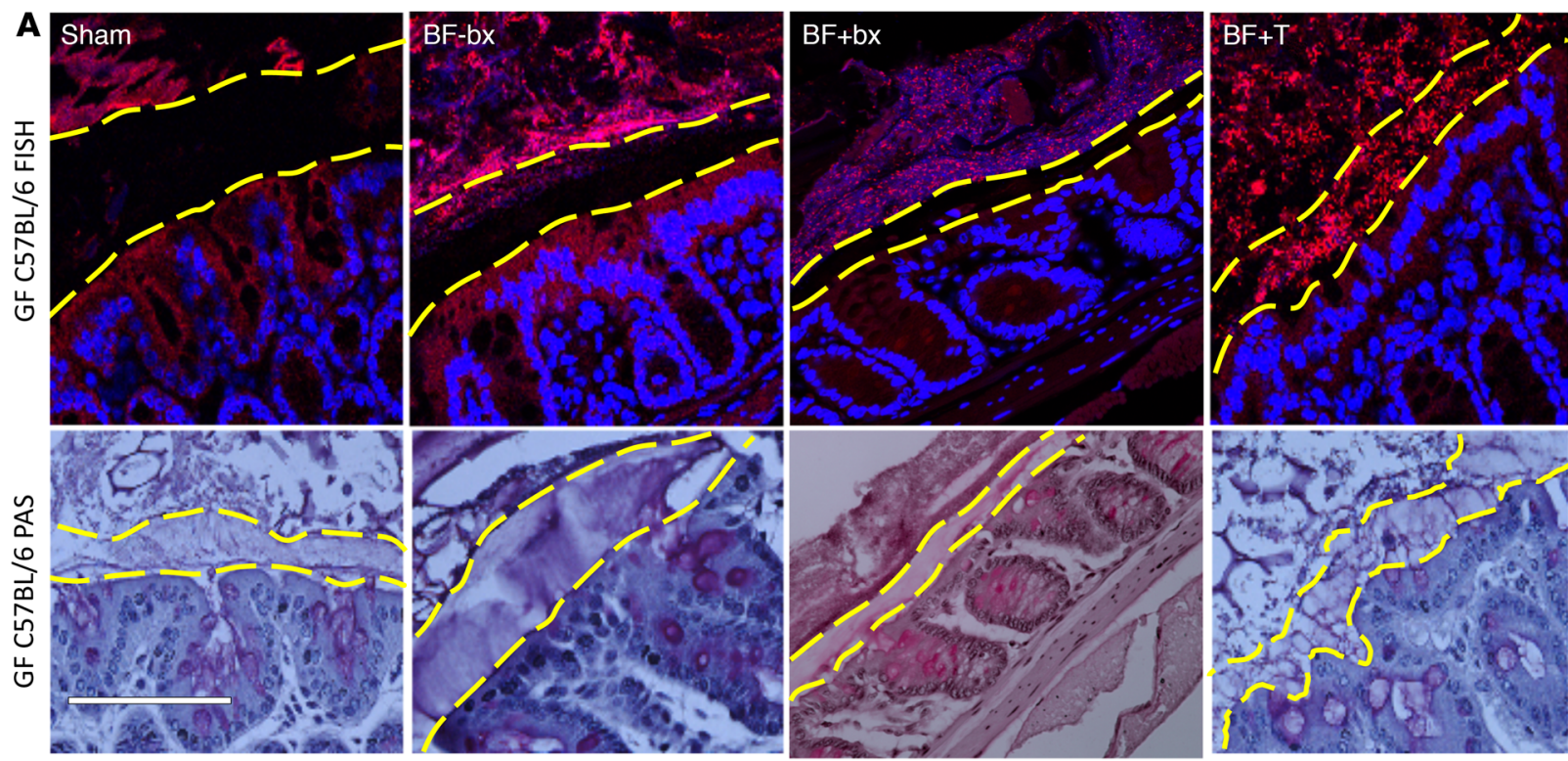

Stained with all bacterial probe, red fluorescence, top panels.

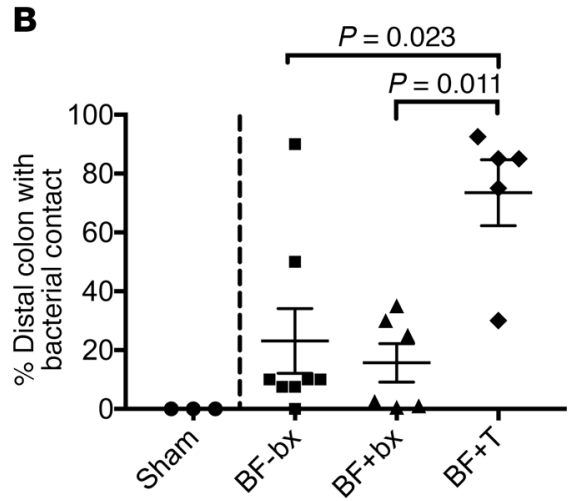

Figure 6. Human $\mathrm{BF}^{+}$tumor tissue inocula induce rapid formation of colon biofilms in mice. (A) Biofilm analysis of distal colon of GF C57BL/6 mice inoculated for 1 week with $\mathrm{BF}^{-}$or $\mathrm{BF}^{+}$ human colonoscopy biopsy inocula or human colon mucosal BF+T inoculum. Representative images from $n=3$ (sham); $n=8$ (BF-bx); $n=6$ (BF+bx); $n=5$ (BF+T). Top panels, FISH staining using all bacterial probe (red fluorescence, see Methods); bottom panels, PASstained sections show mucus staining. The inner mucus layer is outlined in yellow dashed lines in upper and lower panels. Only the human BF+T inoculum consistently induced robust bacterial invasion in the inner mucus layer (biofilm formation) of the distal mouse colon by 1 week. Scale bar: $100 \mu \mathrm{m}$. (B) Quantification of murine (C57BL/6) distal colon bacterial/ epithelial cell contact from Figure 6A. Data are displayed as mean \pm SEM and were analyzed by Mann-Whitney $U$ test. $P<0.025$ was considered significant with Bonferroni's correction. Shams are presented for completeness, but were not included in the statistical analysis.

copy biopsy) or biofilm-negative (colonoscopy biopsy) mucosa. A probe set designed to recognize the major taxa (Lachnospiraceae, Fusobacterium, Bacteroidetes, and Proteobacteria; ref. 15) present in the biofilm inocula was utilized for this analysis. All polymicrobial biofilm communities, regardless of the source, at least partially invaded and colonized the distal mucus layer of GF $A p c^{\text {Min } 1850 /+}$ and $A p c^{\text {Min } 1850 /+} ; I l 10^{-/-}$mice at 12 weeks after inoculation (Figure 5A). In addition, the major phyla from the biofilm inocula were detected in each mouse group and confirmed to make up the majority of bacteria invading the mucus, with the exception of Fusobacterium (Figure 5A). Fusobacterium were not detected in the mucosa of any mouse colon, despite being abundant in the original biofilm-positive tumor inoculum. Further, colon epithelial cell invasion of biofilm members (multiple phyla) was detected in mice from the biofilm-positive inoculation groups (Figure 5B). In contrast, biofilm-negative colonoscopy biopsy inocula did not induce abundant mucus invasion and were marked by a thicker, more intact mucus layer on the murine colon (Figure 5C and Supplemental Figure 5).

To determine whether murine biofilm assembly occurs rapidly after mouse inoculation with human colon mucosal biofilms, GF C57BL/6 mice were inoculated with biofilm-negative healthy subject biopsies, biofilm-positive healthy subject biopsies, or biofilm-positive tumor inocula followed by distal colon tissue fixation with Carnoy's and analysis for distal colon biofilms by FISH 1 week later. At this early time point, only the biofilmpositive tumor inoculum induced murine colon biofilms ( 5 of 5 $\mathrm{BF}+\mathrm{T}$ vs. 0 of $8 \mathrm{BF}-\mathrm{Bx}, 0$ of $6 \mathrm{BF}+\mathrm{Bx}$, and Oof 3 sham mice; Figure $6 \mathrm{~A}$ ). Overall, at least $70 \%$ of the distal colon mucosa of mice inoculated with biofilm-positive tumor inoculum versus approximately $20 \%$ in mice inoculated with biofilm-negative biopsy inoculum displayed invasive bacteria visible in the mucus layer with epithelial cell contact (Figure 6B, $P=0.023$ ). However, in none of the mice inoculated with biofilm-negative biopsy inoculum did this limited invasion constitute either the required density or length for a biofilm (see Methods). Thus, assembly of biofilm communities on murine colon tissue occurs rapidly after inoculation only with human biofilm-positive tumor bacterial communities. Further studies with longer time courses will be necessary to determine whether biofilm-positive healthy biopsy communities can invade colon mucus in mouse models.

Human colon biofilm status is associated with microbiota changes

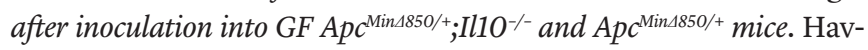
ing shown that biofilm-positive status directly influences colon carcinogenesis in mice, we analyzed the microbiome to gain further insight into the communities associated with this phenotype. We 
A
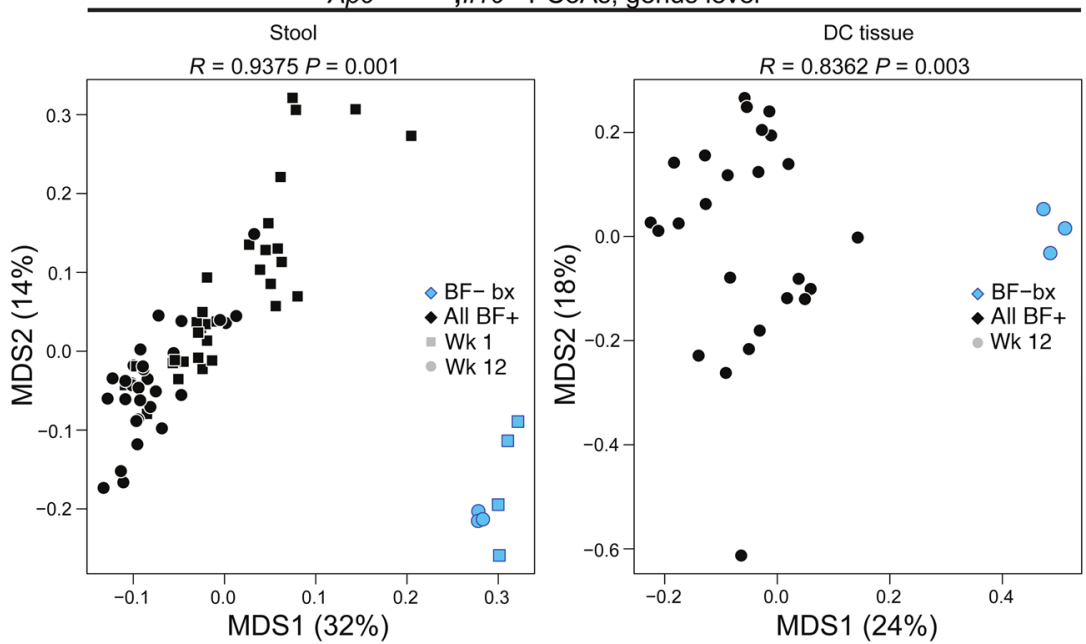

B
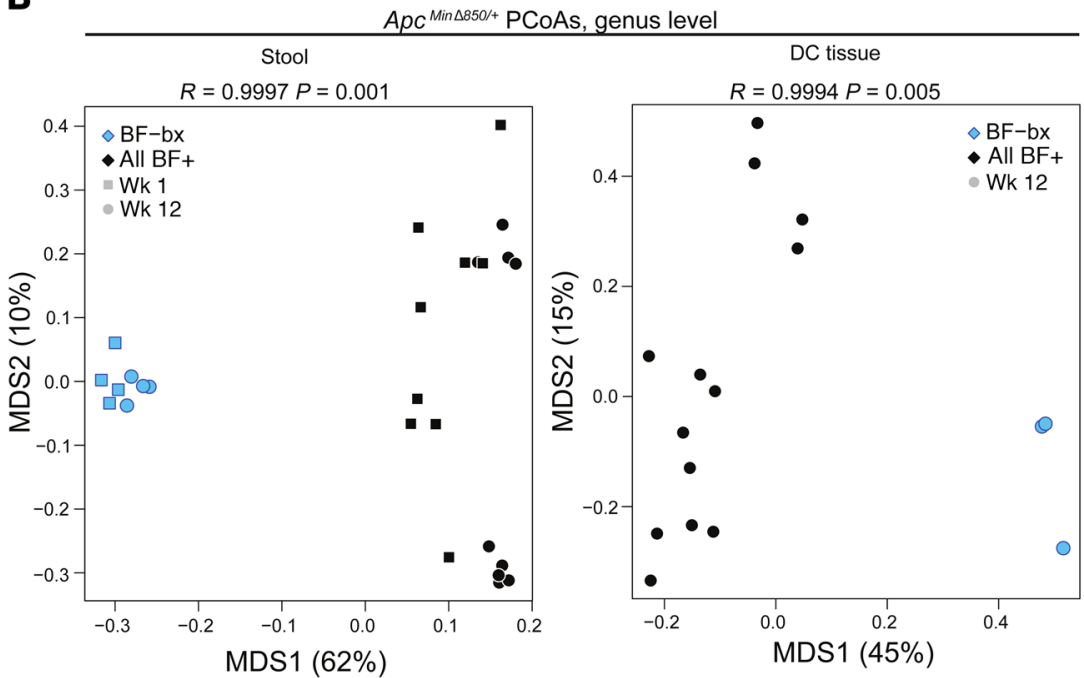

Figure 7. Human colon tissue biofilm status is associated with distinct microbiota changes in GF Apc ${ }^{\mathrm{Min} 1850 /+;} ; \| 10^{-/-}$and $\mathbf{A p c}^{\mathrm{Min} 1850 /+}$ mouse models after gavage. (A and $\mathbf{B}$ ) Genus level PCoAs of $A p c^{M i n} 1850 /+; / 110^{-/-}$stool and distal colon tissue microbiota (A) and $A p c^{M i n} 4850 /+$ mouse stool and distal colon tissue microbiota $(\mathrm{B})$. The $3 \mathrm{BF}^{+}$groups $(\mathrm{BF}+\mathrm{bx}, \mathrm{BF}+\mathrm{NF}$, and $\mathrm{BF}+\mathrm{T}$ ) are combined into 1 group denoted $\mathrm{BF}^{+}$. Numbers in parentheses indicate the percentage of variation explained by that axis. The different symbols represent the 1-week (squares) and 12-week (circles) time points for the stool samples. inoculum group except the $A p C^{\text {Minus50/+} ; I I 10^{-/-}}$ biofilm-positive tumor group (Supplemental Figure 7). Strikingly, an analysis of similarities (ANOSIM) that explicitly incorporated temporal variation still reported striking differences between biofilm-negative and biofilm-positive groups in both $A p c^{\text {Min } 1850 /+} ; I_{11 O^{-/-}}$and $A p c^{\text {Min } 1850 /+}$ mice (Figure 7, A and B). Importantly, although the amount of bacteria differed between the 4 initial inocula, overall bacterial levels normalized quickly in inoculated mice, with no significant difference at 1 week between the groups in stools or tissues as assayed by quantitative PCR (qPCR) for the universal bacterial 16S rRNA gene $(P=0.091$ and $P=0.8349$, respectively; Supplemental Figure 8A). Thus, the differences in tumorigenesis according to biofilm status at 12 weeks (Figure 1) are most likely due to differences in microbiota composition rather than overall changes in bacterial levels between the groups of mice.

In addition to compositional differences, biofilm-negative-associated $\mathrm{ApC}^{\mathrm{Min} 8850 /+} ; \mathrm{Il1O}^{-/-}$ mice had decreased Shannon diversity and richness $(P<0.005)$ in the stool and decreased Shannon diversity $(P=0.0009)$ in the distal colon tissue compared with mice inoculated with biofilmpositive human mucosal tissues (Supplemental Figure 8B). Similarly, Apc $\mathrm{Cin}^{\text {M850/+ }}$ mice inoculated with biofilm-negative tissues had decreased richness $(P=0.0456)$ in the stool and decreased Shannon diversity $(P=0.0359)$ in distal colon tissue compared with biofilm-positive-inoculated $A p c^{\text {Min } 1850 /+}$ mice (Supplemental Figure 8C).

Twenty-four genera in $\mathrm{ApC}^{\text {Min } 1850 /+} ; \mathrm{IllO}^{-/-}$ (Figure 8A) and 14 genera in $\mathrm{Apc}^{\mathrm{Min} 4850 /+}$ mice (Figure $8 \mathrm{~B}$ ) were significantly different between the biofilm-negative- and biofilm-positive-inoculated groups. Eleven genera were significant in both genotypes; 6 of these were significantly increased in both the stool and distal colon tissue compartments of biofilm-positive inoculated mice in both mouse genotypes (Clostridium XVIII, Erysipelotrichaceae incertae used 16S rRNA amplicon sequencing to determine the microbial composition in the original human biofilm-positive and biofilmnegative inocula as well as in the stool samples (1 and 12 week) and end point (12 week) distal colon tissues collected from the inoculated GF ApC $C^{\text {Min } 1850 /+} ; I_{11 O^{-/-}}$and $A p C^{\text {Min } 1850 /+}$ mice. We used samples from GF mice so that these analyses would not be confounded by murine microbiota. PCoA analysis demonstrated a clear separation between the original human mucosal inocula and both the murine stool and distal colon murine tissues, suggesting that a major restructuring of the inoculated human microbial communities occurred in the mouse colon (Supplemental Figure 6, A and B). Longitudinal fecal analysis revealed progressive changes in microbiota composition, with 1-week stool samples clustering apart from 12 -week samples in every murine sedis, Escherichia/Shigella, Eubacterium, Parabacteroides, and Robinsoniella) (Figure 8, A-C and Supplemental Table 2).

Phylogenetic Investigation of Communities by Reconstruction of Unobserved States (PICRUSt) (25), which infers metagenomics function from 16S rRNA amplicon-sequencing data, suggested the biofilm-positive-associated mice had a stool microbiota with a greater capacity to invade epithelial cells and synthesize peptidoglycan (Supplemental Figure 8D). Both of these bacterial functions may contribute to biofilm formation (11). To confirm these data, we performed RNA-Seq on mucosa from biofilm-positive tumor- and biofilm-negative biopsy-associated Apc $\mathrm{Cin}^{\mathrm{M} 850 /+}$ mice to diminish the impact of the enhanced inflammation observed in $\mathrm{Apc}^{\mathrm{Min} 1850 /+} ; \mathrm{Il10^{-/- }}$ mice. Our metatranscriptome analysis (see 
A

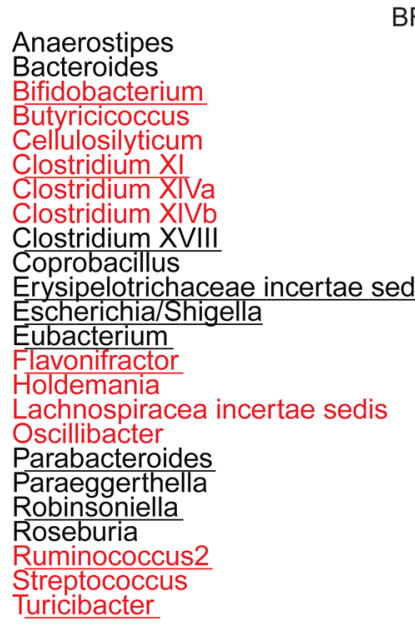

B

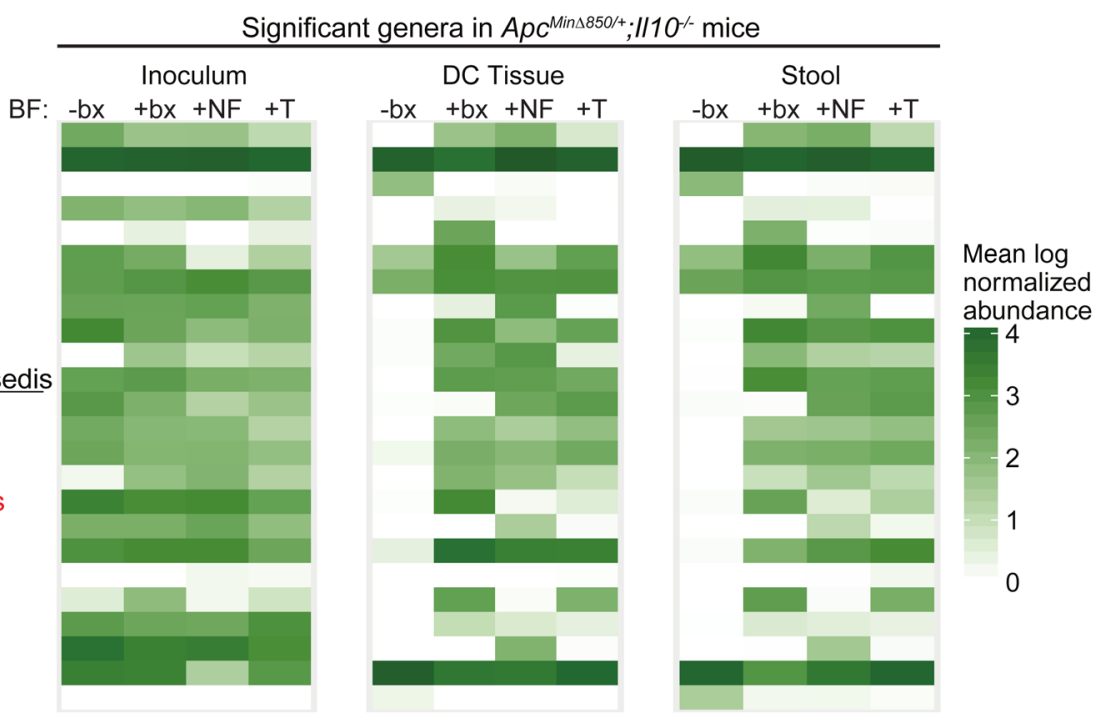

Significant genera in $A p c^{M i n \Delta 850 /+}$ mice

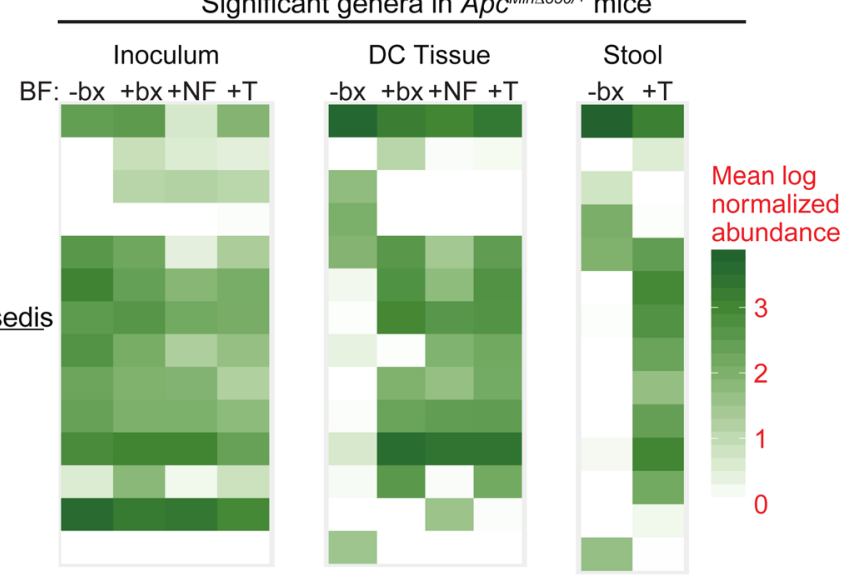

C

Mean $\log _{10}$ normalized relative abundance levels of top significant genera in $A p C^{\text {Min } \Delta 850 /+;} ; 1 / 10^{-/}$stool
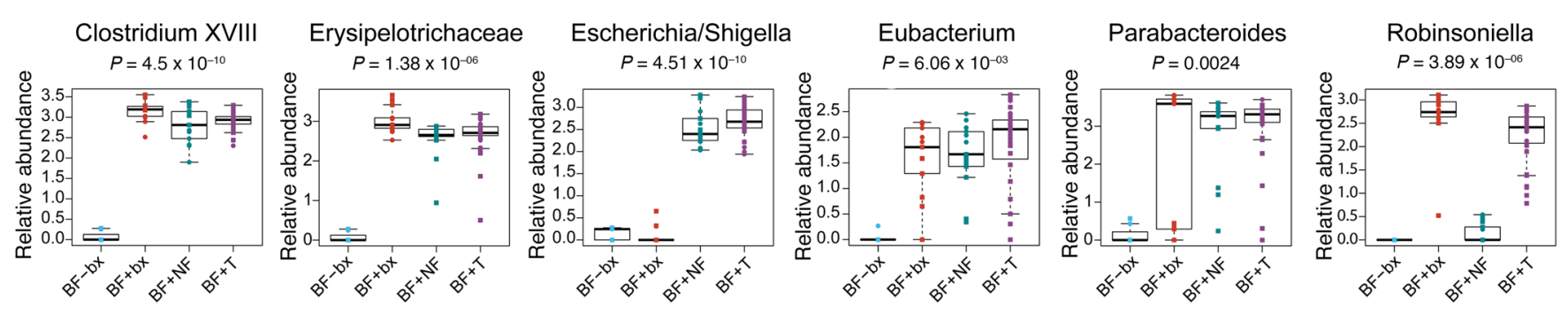

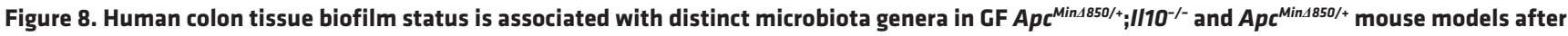
gavage. (A and B) Heatmaps depicting mean $\log _{10}$ normalized relative abundances of all genera that were significantly different according to biofilm status

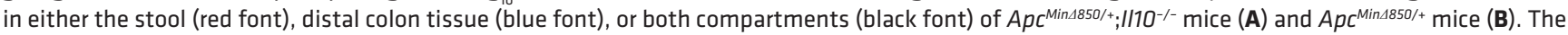
11 genera common to both genotypes are underlined. Corresponding $\log _{10}$ normalized relative abundances of each inoculum are shown for comparison. (C) Box plots of the mean $\log _{10}$ normalized relative abundances of individual genera in $A p c^{M i n} 1850 /+; / 110^{-/-}$stool that were significantly different in both the stool and distal colon compartments in both genotypes. All $P$ values are Benjamini-Hochberg adjusted.

Methods and Supplemental Table 3) confirmed that microbial genes associated with bacterial invasion of epithelial cells and peptidoglycan biosynthesis were differentially upregulated in the mucosa of mice associated with human biofilm-positive tumor relative to biofilm-negative biopsy (Supplemental Figure 9). The analysis further revealed increased differential expression of microbial genes associated with bacterial secretion systems, cytoskeletal pro- teins, novobiocin synthesis, and sporulation in the mucosa of mice associated with human biofilm-positive tumor inocula.

In summary, biofilm-positive-associated mice have an altered microbiota and display an increased capacity to perform biofilmrelated functions, suggesting there are specific biofilm-associated bacteria and bacterial activities that promote tumorigenesis, consistent with our in vivo murine results (Figure 1). 


\section{Discussion}

Genomic studies to date using mucosal tissues or feces from CRC patients and healthy subjects highlight the polymicrobial nature of the interaction between intestinal host cells and microorganisms in CRC development $(26,27)$. Although 2 studies have attempted to test the functional impact of microorganisms present in feces on colon carcinogenesis in murine models $(12,13)$, the biological contribution of microorganisms present in mucosal tissues of CRC patients has not been previously reported. Moreover, since the presence of bacterial biofilms is associated with CRC $(11,15,22)$, it is important to understand the functional role of colon mucosal bacterial organization in CRC. In this study, we observed that direct inoculation of human biofilm-positive mucosal tissue communities obtained from either healthy or sporadic CRC patients promoted tumorigenesis in 3 different genetic murine models of CRC (GF Apc $c^{\mathrm{Min} \Delta 850 /+} ; \mathrm{IllO}^{-/-}, \mathrm{GF} A p \mathrm{c}^{\mathrm{Min} \Delta 850 /+}$, and SPF Apc Min $1716 /+$ ). Further, colon carcinogenesis was transmitted to additional GF $A p c^{\text {Min } 1850 /+} ; \mathrm{IllO}^{-/-}$mice by mucosal murine tissue colonized with microbes derived from human biofilm-positive tumor, but not biofilm-negative biopsy, communities. Two previous studies examining the functional role of CRC-associated fecal microbial communities suggested that community composition (13) and health status of the host (12) contribute to tumorigenesis in chemical CRC models. Our results using genetic CRC models suggest an additional contributing factor, the organization of the microbiota at the mucosal surface. Of particular interest was our observation that even the biofilm-positive, mucosal-associated microbial communities pooled from healthy patients promoted tumorigenesis in our preclinical models. These findings suggest that the composition and organization of the microbiota that established in mice, rather than the health status of the human donor, are associated with tumor development. This is clearly supported by our observation that, although biofilm-negative mucosal-associated microbial communities rapidly proliferated to the level observed with biofilm-positive, mucosal-associated communities and induced histologic intestinal inflammation in $\mathrm{Apc}^{\mathrm{Min} \Delta 850 /+} ; \mathrm{IllO}^{-/-}$mice, these mice failed to develop CRC.

One limitation of our approach is that the carcinogenic potential of the biofilm communities tested cannot be ascribed to colon biofilm formation in an individual patient. Limited material (particularly from colonoscopy biopsies) and the critical importance of demonstrating reproducibility of the results in this initial work made individual patient analyses not possible. Nonetheless, these data indicate that human colon mucosal tissues with biofilms, even in the healthy individual undergoing screening colonoscopy, contain carcinogenic bacteria. Critical, but challenging, next steps include microbiologic recovery of mucosal biofilm communities and functional analyses to identify specific carcinogenic bacteria and/or consortia. This knowledge will inform human epidemiologic studies to identify the relationship between harboring these bacteria (in either the mucosa and/or stool) and disease state in individual patients (presence or absence of colon polyposis or CRC). We anticipate that defining these bacteria or consortia will advance approaches to identifying those at risk for colon carcinogenesis, a goal of increasing importance with recognition of the expanding numbers of colon cancer victims globally and in younger populations $(28,29)$.
Additional striking findings were that tumor-associated biofilms rapidly reestablish just 1 week after inoculation into GF WT C57BL/6 mice and biofilm-positive biopsy and tumor-associated communities induce colon mucosal inflammation, particularly IL-17, in both WT and $A p c^{\text {Min } 1850 /+}$ mice. IL-17 has previously been implicated in contributing to tumorigenesis in ETBF-colonized $(4,21)$ and $\mathrm{ETBF} / p k s^{+}$E. coli-cocolonized mice (22) and is known to adversely affect the prognosis of CRC patients (30). Together, these data support a procarcinogenesis interplay between specific types of microbes and IL-17-associated inflammation during CRC development. Our results demonstrate a significant correlation between histologic distal colon inflammation and tumor formation induced by biofilm-positive communities in $\mathrm{Apc}^{\mathrm{Min} \Delta 850 /+} ; \mathrm{IllO}^{-/-}$ mice, which are genetically prone to developing colitis in the presence of microbes (20), and further highlight the contribution of inflammation to colon tumor initiation and progression. The rapid induction of inflammation and reestablishment of microbes from human biofilm-positive tissues into murine biofilms may partly explain why tumors developed so quickly (just 3 weeks after association) in the SPF $A p c^{\mathrm{Min} \Delta 716 /+}$ mice. Alternatively, the better developed immune system of the SPF mouse and/or unexpected interactions of human and murine microbes, compared with the GF mouse, may have contributed to this early carcinogenesis. These findings suggest biofilm-positive microbial communities have carcinogenic properties that are retained and rapidly induce tumorigenesis upon introduction into susceptible mice.

Multiprobe FISH analysis of sporadic CRC patients demonstrated Bacteroidetes, Lachnospiraceae, Fusobacterium, and Proteobacteria taxa within the mucosal-associated bacterial biofilms within CRC patients $(11,15)$. Each of these taxa except Fusobacterium was represented in the biofilms of biofilm-positive-

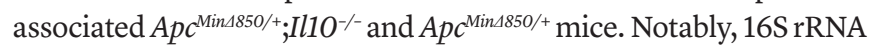
amplicon sequencing detected Fusobacterium in the stools of GF

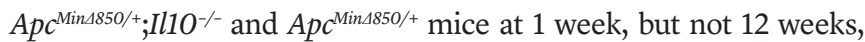
after inoculation. Attempts to recover Fusobacteria from our frozen human CRC samples to date have been successful in only 1 instance (C.L. Sears, B. Ellis, and S. Bullman, unpublished observations). In prior work, Fusobacteria were primarily detected in CRC-associated mucosa and were infrequently associated with normal colon mucosa from either CRC patients or healthy individuals undergoing colonoscopy $(11,15,31,32)$. Collectively, these data indicate that the biofilm formation and early colon tumorigenesis in our mouse models did not require Fusobacteria and support recent data suggesting a role for Fusobacteria in late-stage human CRC disease (6). Consistent with this conclusion, human CRC-derived strains of F. nucleatum were not carcinogenic in GF models of colon carcinogenesis (20).

Previously, invasion into the host epithelium was observed in biofilm-covered tumors and paired normal mucosal tissues from human CRC patients $(15,22)$ and again was observed in most GF biofilm-positive-associated mice. In contrast, biofilm-negativeassociated $A p \mathrm{CMin}^{\mathrm{Mi50/+}} ; \mathrm{IllO}^{-/-}$mice displayed limited mucus bacterial invasion and epithelial cell contact after 12 weeks (Figure $5 \mathrm{C}$ ), but this observation may relate to the nature of the $\mathrm{Il}^{-/-}$ model after colitis develops. Indeed, previous FISH analyses of $\mathrm{Il} \mathrm{O}^{-/-}$or dextran sulfate sodium (DSS) mice revealed that bacteria adhere to and, in some cases, invaded the mucosa of the distal colon compared with WT mice (23). Mucosal-associated biofilms 
have also been observed in inflammatory bowel disease patient mucosal biopsies (33).

The FISH analysis of biofilm-positive-associated mice suggested these communities establish quickly within mice; this observation is consistent with our 16S rRNA gene-sequencing results, which show stool microbiota separation based on biofilm status 1 week after inoculation. Several of the genera we identified as differentially abundant according to biofilm status in the $\mathrm{ApC}^{\mathrm{Min} 1850 /+} ; \mathrm{Il1O}^{-/-}$and $\mathrm{ApC} \mathrm{Min}^{\mathrm{in} 850 /+}$ mice have been previously associated with human CRC patients. Bifidobacterium has been reported to be depleted in CRC patients $(10,34)$ and was depleted in the mice inoculated with microbes from biofilm-positive tissues. In contrast, Clostridium XI, Clostridium XVIII, Erysipelotrichaceae incertae sedis, Escherichia/Shigella, Eubacterium, and Parabacteroides genera were increased in biofilm-positive-associated mice and have been shown to be increased in CRC patients (10, 34-37). It is still unclear whether these bacteria interact with one another and whether all or just some of these bacteria contribute to CRC development. Synergy between multiple types of bacteria is likely to be one contributing factor, given the recent identification of ETBF and $p k s^{+}$E. coli within familial adenomatous polyposis mucosal biofilms and the demonstration that cocolonizing susceptible mice with both bacteria enhanced tumor formation compared with either bacterium alone (22).

Together our findings suggest both composition and bacterial organization are important factors contributing to CRC development. We anticipate that evaluating composition and bacterial organization as well as the type of inflammatory response will provide a clearer picture of how microorganisms contribute to the initiation and progression of cancer. Identifying candidate procarcinogenic species and communities from human colon mucosal biofilms may enable earlier screening to predict patients at risk of developing CRC and allow application of interventions to disrupt the progression of colon carcinogenesis.

\section{Methods}

Animals. All experiments with 129/SvEv GF Apc ${ }^{\text {Mins850/+ }}$ and $A p c^{\text {Min } 1850 /+}$; $I l 10^{-/-}$multiple intestinal neoplasia (Min) mice were conducted at the University of Florida. GF $A p C^{\text {Min } 1850 /+}$ and $A p C^{\text {Min } 1850 /+} ; \mathrm{IllO}^{-/-}$mice were previously described (20). Experiments using GF C57BL/6 and GF or SPF C57BL/6 Apc $\mathrm{Min}^{\mathrm{in} 716 /+}$ mice were conducted at Johns Hopkins University.

Biofilm evaluation and microscopy. Patient tissues were collected and screened for biofilms, as described previously $(11,15,22)$. In brief, patient tumor $(\mathrm{T})$ and normal flanking (NF, collected from the distal margin of the surgical resection) tissues were collected after surgical resection, and healthy control patient tissues were collected from the right and left colon by colonoscopy biopsy (bx). Part of the patient tissue was fixed in Carnoy's solution (60\% methanol, 30\% acetic acid, and $10 \%$ chloroform) and biofilm status assessed by FISH with a universal bacterial probe (EUB338); bacterial density was quantified with ImageJ (NIH). Sections were stained with periodic acid-Schiff (PAS) to confirm mucus presence and preservation, and successive sections were hybridized with the EUB338 universal bacterial probe. Carnoy's fixed tissues were defined as biofilm positive if there were more than $10^{9}$ bacteria/ml for CRC or tumor patients or more than $10^{8}$ bacteria/ $\mathrm{ml}$ for colonoscopy biopsies invading the mucus layer (within $1 \mu \mathrm{m}$ of the epithelium) for at least $200 \mu \mathrm{m}$ of the epithelial surface. All human colon samples were screened twice in a randomized, blinded manner.

Because in mice, only the distal $3 \mathrm{~cm}$ of the colon contains a striated, nonpermissive mucus layer, at 1 week after infection, the distal 3 $\mathrm{cm}$ of each GF C57BL/6 mouse colon was separated from the remainder of the colon and fixed using Carnoy's solution (38), with residual stool intact to preserve the mucus layer. This distal $3 \mathrm{~cm}$ region was screened by FISH with the EUB338 all-bacterial probe and DAPI for the relative percentage of the entire distal colonic epithelium that was in direct contact (within $1 \mu \mathrm{m}$ ) with bacteria. Each mouse colon was scored twice in a blinded, randomized manner, with the 2 scores averaged to obtain a final percentage for each mouse. Data presented are representative of 2 separate experiments, one with shams, BF-Bx, and $\mathrm{BF}+\mathrm{T}$, and a second with shams, $\mathrm{BF}-\mathrm{Bx}$, and $\mathrm{BF}+\mathrm{Bx}$. PAS stains of a sequential slide section were used to confirm the presence of mucus.

Human colonic biofilms were imaged as previously described (15). Confocal images of FISH-stained mouse colons were taken on a Zeiss LSM 780 META laser scanning microscope at $\times 400$ with LSM Zen imaging software with linear unmixing in the Johns Hopkins University School of Medicine Microscope Core Facility, unmixed using Zen software, and finally merged in ImageJ. PAS-stained mouse colons were imaged on a Nikon Eclipse E800 microscope with a Nikon DXM1200 camera.

Human tissue inocula preparation. The study design was developed to give priority to reproducibly testing using multiple murine models the hypothesis that human mucosal microbiota populations could induce colon carcinogenesis. Thus, to allow for limitations in available human tissues and GF mice, we used inocula composed of tissue from 5 different patients (Supplemental Table 1). Biofilm negative $\left(\mathrm{BF}^{-}\right)$and positive $\left(\mathrm{BF}^{+}\right)$inocula were prepared from $3 \mathrm{~mm}$ diameter tissue pieces that were collected from healthy or CRC patients, snap-frozen, and stored at $-80^{\circ} \mathrm{C}$. The BF-bx and $\mathrm{BF}+\mathrm{bx}$ inocula were pooled from separate groups of healthy patients, while the $\mathrm{BF}+\mathrm{NF}$ and $\mathrm{BF}+\mathrm{T}$ inocula were from the same set of CRC patients except in one instance (Supplemental Table 1). All inocula were prepared anaerobically by mincing and homogenizing tissue pieces in PBS in an anaerobic hood to a final dilution of 1:20 (weight/volume).

GF and SPF mouse colonization. Six- to fourteen-week-old GF 129/ SvEv Apc $\mathrm{Min} \triangle 850 /+^{+}$and $A p c^{\text {Min } 1850 /+} ; \mathrm{IllO}^{-/-}$mice (males and females) (University of Florida) were transferred to gnotobiotic isolators (separate isolator for each experimental group) and gavaged with 100-200 $\mu \mathrm{l}$ of inoculum. Mice were euthanized at indicated time points, and colonoscopy was performed as described previously (39) before sacrifice. The small intestine, cecum, and colon were cut open longitudinally, and macroscopic tumors were counted. About 1 to $2 \times 0.5 \mathrm{~cm}$ snips were taken from the proximal and distal colon, flash-frozen in liquid nitrogen, and stored at $-80^{\circ} \mathrm{C}$ until analysis. The rest of the colon was Swiss-rolled and fixed in $10 \%$ neutral buffered formalin solution. Swiss rolls were processed, paraffin-embedded, sectioned, and stained with H\&E by the Molecular Pathology Core at the University of Florida. Histological scoring of inflammation was performed blindly by 2 investigators, as described previously (5). Whole colon inflammation scores were calculated as the average between the proximal and distal colon region scores, while distal colon inflammation scores represent the inflammation score in the distal colon region.

Murine reassociation experiments utilized 6- to 13-week-old GF $129 / \mathrm{SvEv} A p C^{\operatorname{Min} 1850 /+} ; \mathrm{IllO}^{-/-}$mice. For these mice, the inocula were anaerobically homogenized murine colon tissues of 4 mice originally 
inoculated with human BF-bx (for 12 weeks) or $\mathrm{BF}+\mathrm{T}$ (for 16-20 weeks) inocula. Flash-frozen murine tissues were stored at $-80^{\circ} \mathrm{C}$ until anaerobic inocula preparation. Once inocula were prepared, they were stored at $-80^{\circ} \mathrm{C}$ until administration into mice as described above.

The 1-week association experiment for flow cytometry analysis with GF $A p c^{\mathrm{Min} 1850 /+}$ mice was completed in 2 mini-isolators (1 per inoculation group; see Results). For this experiment, the BF-bx inoculum was diluted 1:2, while the BF+T inoculum was diluted 1:4. Each mouse received 100 $\mu \mathrm{l}$ of the diluted inoculum, and mice were euthanized 1 week after inoculation. Colons were shipped overnight on ice to Johns Hopkins University, where flow cytometry analysis (below) was performed.

GF C57BL/6 mice (Johns Hopkins University) were similarly housed and inoculated, as indicated in Results, using individual isolators ( 1 isolator per condition), followed by mouse colon harvest after 1 week for biofilm analysis or flow cytometry, as described below. Prior to inoculation of approximately 6-week-old male and female SPF $A p c^{\text {Min } 4716 /+}$ mice (Johns Hopkins University), mice were given water containing $500 \mathrm{mg} / \mathrm{l}$ cefoxitin for 48 hours. After removal of antibiotic water for 24 hours, mice were inoculated by oral gavage with human colon mucosal homogenates as indicated.

Lamina propria lymphocyte isolation. Colon lamina propria lymphocyte (LPL) isolation has been previously described (21). Briefly, colons were flushed, minced, and digested using $400 \mathrm{U} / \mathrm{ml}$ liberase and 0.1 $\mathrm{mg} / \mathrm{ml}$ DNAase1 (Roche Diagnostics). Leukocytes were isolated by $60 \% / 80 \%$ Percoll gradient separation (GE Healthcare Life Sciences).

Flow cytometry. Staining and flow cytometry analysis have been previously detailed (22). Briefly, LPL were stained for 30 minutes with the LIVE/DEAD Fixable Aqua Viability Stain (Thermo Fisher Scientific) and then washed. Fluorochrome-conjugated antibodies were added for cell-surface staining of lymphoid and myeloid cell subsets and incubated 30 minutes on ice. Stained cells were then washed and analyzed using an LSRFortessa flow cytometer (BD Bioscience) and BD DIVA software (TriStar Inc.). In some experiments, analysis of IFN- $\gamma-$ and IL-17-producing cells was performed using intracellular cytokine staining (ICS), following a 3.5-hour in vitro stimulation of LPL in the presence of eBioscience Stimulation Plus Protein Transport Inhibitor Cocktail (PMA, ionomycin, Brefeldin A, Monensin; Thermo Fisher Scientific). IFN- $\gamma$, IL-17, and Foxp3 staining were performed after fixation of cell surface-stained cells using the Foxp3 Fixation/ Permeabilization Kit (Thermo Fisher Scientific), as previously described (21). Mucosaldendriticcellswere defined as CD11 $\mathrm{c}^{\text {hi }}$ MHC II ${ }^{\text {hi }}$

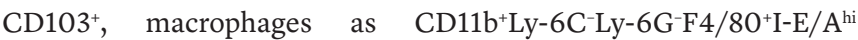
$\left(\mathrm{M} \phi-\mathrm{I}-\mathrm{E} / \mathrm{A}^{\mathrm{hi}}\right)$ or I-E/A $\mathrm{A}^{\text {lo }}\left(\mathrm{M} \phi-\mathrm{I}-\mathrm{E} / \mathrm{A}^{\mathrm{lo}}\right)$, inflammatory $\mathrm{MO}$ as $\mathrm{CD} 11 \mathrm{~b}^{\mathrm{H} \backslash \mathrm{hi}}$ Ly6 $\mathrm{C}^{\text {hi }}$ Ly-6G ${ }^{-}$, PMN neutrophils as CD $11 b^{\text {hi }}$ Ly $6 G^{+}$Ly- $6 G^{\text {Lo }}$, Tregs as $\mathrm{CD}^{+} \mathrm{CD}^{+} \mathrm{Foxp}^{+}$, CD4 non-Tregs as $\mathrm{CD}^{+} \mathrm{CD}^{+}{ }^{+} \mathrm{Foxp}^{-}, \mathrm{CD}^{+} \mathrm{T}$ cells as $\mathrm{CD}^{+}{ }^{+} \mathrm{CD} 4^{+}{ }^{+} \mathrm{Foxp}^{-} \mathrm{CD}^{+}, \gamma \delta \mathrm{T}$ cells as $\mathrm{CD}^{+}{ }^{+} \mathrm{CD} 4^{+}{ }^{+} \mathrm{Foxp} 3^{-} \mathrm{CD} 8$ $\gamma \delta \mathrm{TCR}^{+}$, and NKT cells as $\mathrm{CD}^{+}{ }^{+} \mathrm{CD} 4^{+}{ }^{+}$Foxp $3^{-} \mathrm{CD} 8^{-} \gamma \delta \mathrm{TCR}^{-}$. ILCs were defined as CD3-Thy1.2 $2^{\text {hi }}$. The gating strategies have been previously detailed (22).

DNA extractions and 16S rRNA qPCR assay. DNA was extracted from human tissue inocula and biofilm-positive or biofilm-negative inoculated GF $\mathrm{ApC}^{\mathrm{Min} 4850 /+}$ and $\mathrm{ApC}^{\mathrm{Min} 4850 /+} ; \mathrm{IllO}^{-/-}$stools (collected at 1 week and end point) and distal colon tissues (end point) using phenol/chloroform separation followed by DNeasy Blood \& Tissue Kit (QIAGEN). 16S rRNA qPCR was performed on a CFX384 Touch Real-Time PCR Detection System (Bio-Rad) using the SsoAdvanced Universal SYBR Green Supermix (Bio-Rad). The following universal bacteria 16S rRNA gene primers were used: forward, AGAGTTTGATCCTGGCTCAG; and reverse, ACTGCTGCCTCCCGTAGGAG.

16S rRNA amplicon sequencing. The V1-V3 hypervariable region of the 16S rRNA gene was amplified using primer pairs $27 \mathrm{~F}$ (5'-AGAGTTTGATCCTGGCTCAG-3') and 534R (5'-ATTACCGCGGCTGCTGG-3'). Both the forward and the reverse primers contained universal Illumina paired-end adapter sequences as well as unique individual 4- to 6-nucleotide barcodes between the PCR primer sequence and the Illumina adapter sequence to allow multiplex sequencing (Supplemental Table 4). PCR products were visualized on an agarose gel before samples were purified using the Agencourt AMPure XP Kit (Beckman Coulter) and quantified by qPCR with the KAPA Library Quantification Kit (KAPA Biosystems). Equimolar amounts of samples were then pooled and sequenced with an Illumina MiSeq.

$16 S$ rRNA-Seq analysis. Taxonomic ranks were assigned for the forward reads using the ribosomal database project (RDP) classifier (40) done with confidence set to $80 \%$. Reads were grouped by genera, and the counts were normalized and $\log _{10}$ transformed (41) using the following formula:

$$
\log _{10}\left(\frac{R C}{n} \times \frac{\sum x}{N}+1\right)
$$

(Equation 1)

where $R C$ is the read count for a particular genus in a particular sample, $n$ is the total number of reads in that sample, the sum of $x$ is the total number of reads in all samples, and $N$ is the total number of samples. This equation is used to standardize the effect of adding the pseudocount to samples of different sequencing depth. The PCoA was generated from the Bray-Curtis distance of the normalized and $\log _{10}$-transformed counts using the capscale function in the vegan R package $(42,43)$.

Genera significant for biofilm group (BF-bx, $\mathrm{BF}+\mathrm{bx}, \mathrm{BF}+\mathrm{NF}$, or $\mathrm{BF}+\mathrm{T}$ ) were detected using the lme function in the $\mathrm{R}$ nlme package, with the REML method (44) to fit a generalized mixed linear model of the following form: genera $\sim$ group +1 |cage $+\varepsilon$, where genera indicates the $\log _{10}$-normalized abundance of a particular genera, group indicates the biofilm group, and 1/cage indicates that we used the cage as a random effect. We then ran an ANOVA analysis on the above model to generate $P$ values for the biofilm group. We filtered genera absent in more than a quarter of the samples. The $P$ values for cage were calculated using an ANOVA of this model and a model with the cage removed (genera $\sim$ group $+\varepsilon$ ). All $P$ values were then adjusted for multiple hypothesis testing using the method of Benjamini and Hochberg (45). The heatmap was generated using the R function ggplot2 (46). The code and tables used to generate the 16S rRNA-Seq figures can be found at: https://github.com/afodor/biofilm.

We performed 2 additional analyses on the 16S rRNA data, the first utilizing QIIME (47), version 1.9.1, closed reference at 97\% similarity level using the Greengenes reference dataset release 13_8, and the second employing Deblur (48) workflow, version 1.0.3, with the default parameters (using Deblur's default positive and negative reference filtering) and trim length set to 100 bases. Both pipelines showed similar broad separation between the $\mathrm{BF}^{+}$and $\mathrm{BF}-\mathrm{bx}$ samples (Supplemental Figure 6, C and D).

Functional prediction. Sequences were also analyzed by PICRUSt to infer functional content as previously described $(11,25)$. PICRUSt counts associated with functional categories were also normalized to an even total per sample within each data set. Relative contributions of 
higher-level taxa to functional categories were determined using the metagenome_contributions.py in the PICRUSt package.

RNA extraction, rRNA depletion, and RNA-Seq. Total RNA was extracted from frozen $A p c^{\mathrm{Min} 4850 /+}$ proximal colon tissue snips using the mirVana miRNA Isolation Kit with phenol (Thermo Fisher Scientific, catalog AM1560), according to the manufacturer's instructions, with the addition of an approximately 1:1 mix of $1 \mathrm{~mm}$ acid-washed glass beads and $0.1 \mathrm{~mm}$ zirconia beads and a Precellys 24 (Bertin Instruments, catalog EQ03119-200-RD000.0) bead beater for tissue disruption and lysis. Extracted RNA was treated with the Turbo DNA-free Kit (Thermo Fisher Scientific, catalog AM1907) to remove DNA. Quality control, rRNA depletion, and cDNA library preparation were performed by the University of Florida's Interdisciplinary Center for Biotechnology Research (ICBR) Gene Expression and Genotyping core using the Agilent 2100 Bioanalyzer (Agilent Genomics, catalog G2939BA), Ribo-Zero Gold rRNA Removal Kit (Epidemiology) (Illumina, catalog MRZE724), and the ScriptSeq, version 2, RNASeq Library Preparation Kit (Illumina, catalog SSV21124) starting with $1 \mu \mathrm{g}$ total RNA. Samples were sequenced by the University of Florida ICBR NextGen DNA Sequencing core on the Illumina HiSeq 3000 ( $2 \times 100$ run), multiplexing each sample into 3 lanes to avoid lane effect.

Metatranscriptome analysis. Trimmomatic was used to quality filter and trim the RNA-Seq reads, followed by alignment to the iGenome Mus musculus Ensembl GRCm38 reference genome using BWA, version 0.7.16a; reads with alignments to the host genome were excluded from further evaluation. The remaining set of unaligned reads was next assessed for rRNA and tRNA transcripts by aligning (BWA) to a collection of NCBI rRNA and tRNA sequences and the SILVA rRNA database; reads with rRNA and tRNA alignments were also excluded from further evaluation. Protein-coding geneexpression quantification and annotation were performed by aligning the remaining reads to the human gut microbiome integrated gene catalog (IGC) (49) using Bowtie2 (version 2.3.4.2) followed by quantification using featureCounts from the subread package, version 1.5.3. Differential expression analysis was performed using edgeR; a gene was considered significant if its FDR-adjusted $P$ value was less than 0.05 .

Data availability. 16S rRNA sequences were uploaded to the NCBI's Sequence Read Archive (SRA PRJNA423288). RNA-Seq data were deposited in the NCBI's SRA (PRJNA510126).

Statistics. Statistics for 16S rRNA and RNA-Seq data are described in the above analysis sections. Flow cytometry data were analyzed by 2-way ANOVA with correction for multiple comparisons (Tukey's test). Fisher's exact test was used to analyze proportions and Spearman's rank correlation to evaluate the statistical dependence between the rankings of 2 variables. The log-rank (Mantel-Cox) test and the logrank test for trend were used for survival analyses. All other data were analyzed using the nonparametric unpaired, 2-tailed Mann-Whitney $U$ test for 2-group comparisons or the Kruskal-Wallis test for 3 or more group comparisons as labeled for each figure. Data are presented as mean \pm SEM unless otherwise stated. $P<0.05$ was considered statistically significant except, as indicated in the text or figure legends, when Bonferroni's correction was applied for multiple comparisons. Within figures, the NS label designates $P \geq 0.05$.

Study approval. Animal experiments performed at the University of Florida were approved by the University of Florida Institutional Animal Care and Use Committee, and animal experiments performed at Johns Hopkins University were approved by the Johns Hopkins Uni- versity Institutional Animal Care and Use Committee. Patient tissues were collected as described previously $(11,15,22)$. This study was approved by the Johns Hopkins Institutional Review Board. All samples were obtained in accordance with the Health Insurance Portability and Accountability Act (HIPAA).

\section{Author contributions}

ST, CMD, CJ, and CLS conceived and designed the study. ST, CMD, PF, FH, JLD, LC, RAA, SB, HD, XW, JLP, JG, EPC, XS, MM, XL, JRW, and YY acquired data. ST, CMD, KW, FH, JLD, LC, RZG, RAA, SB, DMP, AAF, JRW, CJ, and CLS analyzed and interpreted data. CMD, CLS, ST, KW, FH, AAF, and CJ drafted the manuscript. ST, CMD, KW, FH, RZG, SW, AAF, JRW, HW, and CLS performed statistical analysis. CJ, CLS, and FH obtained funding. CLS and CJ supervised the study. All authors reviewed and approved the manuscript.

\section{Acknowledgments}

This research was supported by NIH grants R01DK073338, R01DK47700, and R21 CA195226 (all to University of Florida); R01CA151393, R01CA196845, P50 CA62924, P30 CA006973, P30 DK089502, and K99 CA230192 (all to Johns Hopkins University School of Medicine). This work was also supported by NIH Shared Instrumentation grant S10OD016374 for the Zeiss 780 LSM confocal in the Johns Hopkins University Microscope Facility. Additional support was provided by the Bloomberg Philanthropies, the Johns Hopkins Discovery Award, the Commonwealth Foundation, and the Cancer Research Institute/Fight Colorectal Cancer Coalition (Johns Hopkins University School of Medicine) as well as University of Florida Health Cancer Center Funds and the University of Florida Department of Medicine Gatorade Fund. YY is supported by the Crohn's \& Colitis Foundation of America (CCFA) research fellowship award (CCFA, reference 409472). The funders had no role in study design, data collection and analysis, decision to publish, or preparation of the manuscript. The authors are grateful to the germ-free services division of University of Florida's Animal Care Services and the Johns Hopkins University Germ-Free facility for assistance with gnotobiotic experiments. We thank Danielle Ferraguti for her assistance with the DNA extractions and 16S rRNA library preparation. We would also like to thank the University of Florida Molecular Pathology Core for their histology services and the Johns Hopkins University Microscopy Core for assistance. We also thank the University of Florida ICBR Gene Expression and Genotyping and NextGen DNA Sequencing Cores for helping with the RNA-Seq.

Address correspondence to: Cynthia L. Sears, 1550 Orleans Street, CRB2 Building, Suite 1M.05, Baltimore, Maryland 21231, USA. Phone: 410.614.0141; Email: csears@jhmi.edu. Or to: Christian Jobin, 2033 Mowry Road, PO Box 103633, Gainesville, Florida 32611-0882, USA. Phone: 352.294.5148; Christian.Jobin@ medicine.ufl.edu.

CMD's present address is: Food and Drug Administration, Silver Spring, Maryland, USA.

PF's present address is: Vanderbilt University School of Medicine, Nashville, Tennessee, USA. 
1. Sears CL, Garrett WS. Microbes, microbiota, and colon cancer. Cell Host Microbe. 2014;15(3):317-328.

2. Tjalsma H, Boleij A, Marchesi JR, Dutilh BE. A bacterial driver-passenger model for colorectal cancer: beyond the usual suspects. Nat Rev Microbiol. 2012;10(8):575-582.

3. Schwabe RF, Jobin C. The microbiome and cancer. Nat Rev Cancer. 2013;13(11):800-812.

4. Wu S, et al. A human colonic commensal promotes colon tumorigenesis via activation of $\mathrm{T}$ helper type $17 \mathrm{~T}$ cell responses. Nat Med. 2009;15(9):1016-1022.

5. Arthur JC, et al. Intestinal inflammation targets cancer-inducing activity of the microbiota. Science. 2012;338(6103):120-123.

6. Bullman S, et al. Analysis of Fusobacterium persistence and antibiotic response in colorectal cancer. Science. 2017;358(6369):1443-1448.

7. Kumar R, et al. Streptococcus gallolyticus subsp. gallolyticus promotes colorectal tumor development. PLoS Pathog. 2017;13(7):e1006440.

8. Wang X, Yang Y, Huycke MM. Commensalinfected macrophages induce dedifferentiation and reprogramming of epithelial cells during colorectal carcinogenesis. Oncotarget. 2017;8(60):102176-102190.

9. Nakatsu G, et al. Gut mucosal microbiome across stages of colorectal carcinogenesis. Nat Commun. 2015;6:8727.

10. Feng Q, et al. Gut microbiome development along the colorectal adenoma-carcinoma sequence. Nat Commun. 2015;6:6528.

11. Drewes JL, et al. High-resolution bacterial $16 \mathrm{~S}$ rRNA gene profile meta-analysis and biofilm status reveal common colorectal cancer consortia. NPJ Biofilms Microbiomes. 2017;3:34.

12. Wong SH, et al. Gavage of fecal samples from patients with colorectal cancer promotes intestinal carcinogenesis in germ-free and conventional mice. Gastroenterology. 2017;153(6):1621-1633.e6.

13. Baxter NT, Zackular JP, Chen GY, Schloss PD. Structure of the gut microbiome following colonization with human feces determines colonic tumor burden. Microbiome. 2014;2:20.

14. Pelaseyed T, et al. The mucus and mucins of the goblet cells and enterocytes provide the first defense line of the gastrointestinal tract and interact with the immune system. Immunol Rev. 2014;260(1):8-20.

15. Dejea CM, et al. Microbiota organization is a distinct feature of proximal colorectal cancers. Proc Natl Acad Sci U S A. 2014;111(51):18321-18326.

16. Johnson $\mathrm{CH}$, et al. Metabolism links bacterial biofilms and colon carcinogenesis. Cell Metab. 2015;21(6):891-897.

17. Fearon ER, Vogelstein B. A genetic model for colorectal tumorigenesis. Cell.1990;61(5):759-767.

18. Moser AR, Dove WF, Roth KA, Gordon JI. The Min (multiple intestinal neoplasia) mutation: its effect on gut epithelial cell differentiation and interaction with a modifier system. J Cell Biol. 1992;116(6):1517-1526.

19. Kotlarz D, et al. Loss of interleukin-10 signaling and infantile inflammatory bowel disease: implications for diagnosis and therapy. Gastroenterology. 2012;143(2):347-355.

20. Tomkovich S, et al. Locoregional effects of microbiota in a preclinical model of colon carcinogenesis. Cancer Res. 2017;77(10):2620-2632.

21. Housseau F, et al. Redundant innate and adaptive sources of IL17 production drive colon tumorigenesis. Cancer Res. 2016;76(8):2115-2124.

22. Dejea CM, et al. Patients with familial adenomatous polyposis harbor colonic biofilms containing tumorigenic bacteria. Science. 2018;359(6375):592-597.

23. Swidsinski A, Loening-Baucke V, Lochs $\mathrm{H}$, Hale LP. Spatial organization of bacterial flora in normal and inflamed intestine: a fluorescence in situ hybridization study in mice. World J Gastroenterol. 2005;11(8):1131-1140.

24. Johansson ME, Larsson JM, Hansson GC. The two mucus layers of colon are organized by the MUC2 mucin, whereas the outer layer is a legislator of host-microbial interactions. Proc Natl Acad Sci U S A. 2011;108 Suppl 1:4659-4665.

25. Langille MG, et al. Predictive functional profiling of microbial communities using $16 \mathrm{~S}$ rRNA marker gene sequences. Nat Biotechnol. 2013;31(9):814-821.

26. Borges-Canha M, Portela-Cidade JP, Dinis-Ribeiro M, Leite-Moreira AF, PimentelNunes P. Role of colonic microbiota in colorectal carcinogenesis: a systematic review. Rev Esp Enferm Dig. 2015;107(11):659-671.

27. Nistal E, Fernández-Fernández N, Vivas S, Olcoz JL. Factors determining colorectal cancer: the role of the intestinal microbiota. Front Oncol. 2015;5:220.

28. Siegel RL, et al. Colorectal cancer incidence patterns in the United States, 1974-2013. J Nat Cancer Inst. 2017;109(8):djx104.

29. Arnold M, Sierra MS, Laversanne M, Soerjomataram I, Jemal A, Bray F. Global patterns and trends in colorectal cancer incidence and mortal ity. Gut. 2017;66(4):683-691.

30. Tosolini M, et al. Clinical impact of different classes of infiltrating T cytotoxic and helper cells (Th1, th2, treg, th17) in patients with colorectal cancer. Cancer Res. 2011;71(4):1263-1271.

31. Kostic AD, et al. Genomic analysis identifies association of Fusobacterium with colorectal carcinoma. Genome Res. 2012;22(2):292-298.

32. Castellarin M, et al. Fusobacterium nucleatum infection is prevalent in human colorectal carcinoma. Genome Res. 2012;22(2):299-306.

33. Swidsinski A, Weber J, Loening-Baucke V, Hale LP, Lochs H. Spatial organization and com- position of the mucosal flora in patients with inflammatory bowel disease. J Clin Microbiol. 2005;43(7):3380-3389.

34. Chen W, Liu F, Ling Z, Tong X, Xiang C. Human intestinal lumen and mucosa-associated microbiota in patients with colorectal cancer. $P L O S$ ONE. 2012;7(6):e39743.

35. Yu J, et al. Metagenomic analysis of faecal microbiome as a tool towards targeted noninvasive biomarkers for colorectal cancer. Gut. 2017;66(1):70-78

36. Marchesi JR, et al. Towards the human colorectal cancer microbiome. PLOS ONE. 2011;6(5):e20447.

37. Wu N, et al. Dysbiosis signature of fecal microbiota in colorectal cancer patients. Microb Ecol. 2013;66(2):462-470.

38. Hansson GC, Johansson ME. The inner of the two Muc2 mucin-dependent mucus layers in colon is devoid of bacteria. Gut Microbes. 2010;1(1):51-54.

39. Uronis JM, Mühlbauer M, Herfarth HH, Rubinas TC, Jones GS, Jobin C. Modulation of the intestinal microbiota alters colitis-associated colorectal cancer susceptibility. PLOS ONE. 2009;4(6):e6026.

40. Wang Q, Garrity GM, Tiedje JM, Cole JR. Naive Bayesian classifier for rapid assignment of rRNA sequences into the new bacterial taxonomy. Appl Environ Microbiol. 2007;73(16):5261-5267.

41. McCafferty J, et al. Stochastic changes over time and not founder effects drive cage effects in microbial community assembly in a mouse model. ISME J. 2013;7(11):2116-2125.

42. Oksanen JB et al. vegan: Community Ecology Package. https://cran.r-project.org/web/packages/ vegan/vegan.pdf. Accessed February 4, 2019.

43. $\mathrm{R}$ Core Team. R: A language and environment for statistical computing. R Foundation for Statistical Computing, Vienna, Austria. http://www.Rproject.org/. Accessed February 28, 2019.

44. Pinheiro J, Bates D, DebRoy S, Sarkar D, R Core Team. nlme: Linear and Nonlinear Mixed Effects Models. https:/CRAN.R-project.org/ package = nlme. Accessed February 4, 2019.

45. Benjamini Y, Hochberg Y. Controlling the false discovery rate: a practical and powerful approach to multiple testing. J Roy Statist Soc Ser B (Methodological) 1995;57(1):289-300.

46. Wickham H. ggplot2: Elegant Graphics for Data Analysis. New York, NY: Springer-Verlag; 2009.

47. Caporaso JG, et al. QIIME allows analysis of high-throughput community sequencing data Nat Methods. 2010;7(5):335-336.

48. Amir A, et al. Deblur rapidly resolves singlenucleotide community sequence patterns. mSystems. 2017;2(2):e00191-16.

49. Qin J, et al. A human gut microbial gene catalogue established by metagenomic sequencing. Nature. 2010;464(7285):59-65. 